\title{
A FLUX ROPE NETWORK AND PARTICLE ACCELERATION IN THREE-DIMENSIONAL RELATIVISTIC MAGNETIC RECONNECTION
}

\author{
Daniel Kagan ${ }^{1}$, Miloš Milosavljević ${ }^{1}$, and Anatoly Spitkovsky ${ }^{2}$ \\ ${ }^{1}$ Department of Astronomy, University of Texas at Austin, Austin, TX 78712, USA \\ 2 Department of Astrophysical Sciences, Princeton University, Princeton, NJ 08544, USA \\ Received 2012 August 2; accepted 2013 July 2; published 2013 August 14
}

\begin{abstract}
We investigate magnetic reconnection and particle acceleration in relativistic pair plasmas with three-dimensional particle-in-cell simulations of a kinetic-scale current sheet in a periodic geometry. We include a guide field that introduces an inclination between the reconnecting field lines and explore outside-of-the-current sheet magnetizations that are significantly below those considered by other authors carrying out similar calculations. Thus, our simulations probe the transitional regime in which the magnetic and plasma pressures are of the same order of magnitude. The tearing instability is the dominant mode in the current sheet for all guide field strengths, while the linear kink mode is less important even without the guide field, except in the lower magnetization case. Oblique modes seem to be suppressed entirely. In its nonlinear evolution, the reconnection layer develops a network of interconnected and interacting magnetic flux ropes. As smaller flux ropes merge into larger ones, the reconnection layer evolves toward a three-dimensional, disordered state in which the resulting flux rope segments contain magnetic substructure on plasma skin depth scales. Embedded in the flux ropes, we detect spatially and temporally intermittent sites of dissipation reflected in peaks in the parallel electric field. Magnetic dissipation and particle acceleration persist until the end of the simulations, with simulations with higher magnetization and lower guide field strength exhibiting greater and faster energy conversion and particle energization. At the end of our largest simulation, the particle energy spectrum attains a tail extending to high Lorentz factors that is best modeled with a combination of two additional thermal components. We confirm that the primary energization mechanism is acceleration by the electric field in the X-line region. The highest-energy positrons (electrons) are moderately beamed with median angles $\sim 30^{\circ}-40^{\circ}$ relative to (the opposite of) the direction of the initial current density, but we speculate that reconnection in more highly magnetized plasmas would give rise to stronger beaming. Finally, we discuss the implications of our results for macroscopic reconnection sites, and which of our results may be expected to hold in systems with higher magnetizations.
\end{abstract}

Key words: acceleration of particles - instabilities - magnetic fields - magnetic reconnection - plasmas - relativistic processes

Online-only material: color figures

\section{INTRODUCTION}

Magnetic reconnection (e.g., Yamada et al. 2010 and references therein) is of interest in diverse areas of astrophysics, yet its mechanics remain incompletely understood. A tearing instability is thought to be necessary to initiate reconnection in reversing magnetic field configurations, but many astrophysical plasmas have collisional resistivities that are insufficient to facilitate its growth. Interpretations of space plasma measurements (e.g., Chen et al. 2008; Øieroset et al. 2011) and astronomical observations, however, suggest that efficient collisionless reconnection is ubiquitous. Therefore, collisionless effects, which operate on plasma kinetic scales, seem to be required to provide the dissipation necessary for affecting a change of magnetic topology. Magnetohydrodynamic (MHD) models for the dynamics of systems undergoing magnetic reconnection have been available for a long time (e.g., Priest \& Forbes 2000 and references therein). Unfortunately, these models do not describe the underlying nature of (possibly multiscale) plasma organization in the reconnection layer where magnetic energy is being dissipated and the assumptions of ideal MHD do not apply. Understanding the detailed plasma organization on all length scales, from the likely relatively small, plasma kinetic scales, to the potentially much larger scales on which astrophysical dynamical systems "prepare" reconnection sites, and where ideal MHD may be valid, is paramount for completing the theories of a wide variety of astrophysical phenomena and for interpreting space plasma measurements and astronomical observations.

In an effort to develop a picture of magnetic reconnection from first principles, recent particle-in-cell (PIC) simulations have examined the dynamics of reconnection layers that start with a current sheet as thin as the plasma skin depth. PIC simulations of reconnection in pair plasmas have been carried out in two spatial dimensions (Zenitani \& Hoshino 2001, 2007; Jaroschek et al. 2004; Bessho \& Bhattacharjee 2005, 2007, 2010, 2012; Daughton \& Karimabadi 2007; Hesse \& Zenitani 2007; Hoshino 2012) and three dimensions (Zenitani \& Hoshino 2005, 2008; Yin et al. 2008; Liu et al. 2011; Sironi \& Spitkovsky 2011). Among these, several (Zenitani \& Hoshino 2005, 2008; Bessho \& Bhattacharjee 2007; Hesse \& Zenitani 2007; Hoshino 2012) have investigated the role of departure from the idealized, exactly antiparallel reconnection by introducing a perpendicular "guide" field. These various simulations have revealed novel forms of small-scale plasma self-organization that are interesting in their own right, but that must ultimately be related to and embedded within the appropriate larger astrophysical contexts (e.g., Uzdensky et al. 2010). While spacecraft measurements, which can be done in situ, can provide direct clues on how to establish this embedding 
in space plasmas, in extrasolar contexts only an indirect relation can be established between the reconnection process and the observed emission (e.g., Sironi \& Spitkovsky 2011; Cerutti et al. 2012b).

Common features seen in many PIC simulations of magnetic reconnection include the formation of chains of magnetic flux ropes (in three dimensions with a guide field; otherwise, the common terms "islands" or "plasmoids" may still be more appropriate), the merging of smaller flux ropes into larger ones, and an energization of the plasma in the reconnection layer. In three-dimensional simulations, kink-like and oblique modes, as well as secondary instabilities, can impart three-dimensional structure to the reconnection layer.

Typically, the simulations are initialized in the so-called Harris sheet equilibrium describing a current sheet with a thickness similar to the plasma skin depth. The tearing instability first sets in on scales of the initial current sheet thickness. Its nonlinear development produces a chain of skin-depth-scale flux ropes alternating with magnetic X-lines, the three-dimensional generalization of two-dimensional $\mathrm{X}$-points. In X-lines, violation of flux freezing and magnetic line reconnection can be facilitated by a pressure tensor anisotropy (Vasyliunas 1975, see also, e.g., Hesse \& Zenitani 2007 and references therein). Smaller flux ropes tend to merge with each other to form larger ones; this gives rise to magnetic organization on increasingly larger spatial scales. Three-dimensional PIC simulations of guide field reconnection in electron-ion plasmas exhibit these same features, e.g., Daughton et al. (2011) found that oblique modes dominated over tearing modes when the guide field was strong. Additional effects specific to plasmas with an electron-ion mass disparity have also been identified, but are not relevant for the present work.

Energization of particles in reconnection layers has been investigated in a number of PIC simulations (Zenitani \& Hoshino 2001, 2007; Jaroschek et al. 2004; Drake et al. 2006, 2010; Bessho \& Bhattacharjee 2007, 2010, 2012; Egedal et al. 2009; Huang et al. 2010; Oka et al. 2010b; Liu et al. 2011; Sironi \& Spitkovsky 2011; Egedal et al. 2012; Hoshino 2012; Cerutti et al. 2012b). Less attention has been given to particle energization in the general case of reconnection with a guide field in three dimensions (Zenitani \& Hoshino 2008). In two-dimensional simulations, particle acceleration producing a nonthermal energy spectrum, an apparent powerlaw, is often reported. Cerutti et al. (2012b), however, instead detect a new ultrarelativistic thermal component energized by the reconnection.

That the reconnection layers should energize particles is in agreement with analytical considerations (e.g., Speiser 1965; Larrabee et al. 2003; Giannios 2010; Uzdensky et al. 2011; Cerutti et al. 2012a), which find that particles in the vicinity of the X-line in the reconnection layer are accelerated by the nearly-uniform electric field as they repeatedly cross, and are trapped within, the converging plasma flows. Other mechanisms focusing on energetic particles that have moved from the X-line region into the flanking islands have also been suggested (e.g., Drake et al. 2006, 2010). In three-dimensional simulations, evidence for a nonthermal spectrum is less solid. It remains poorly understood which processes limit the energy to which particles can be accelerated in fully dynamical, three-dimensional reconnection layers, and how do the particle energy spectrum, the degree of accelerated particle beaming, and the temporal evolution of the accelerated population depend on the parameters of the reconnection layer.
In this work, we employ three-dimensional PIC simulations to investigate the evolution of current sheets in relativistic pair plasmas undergoing magnetic reconnection. Our simulations add to the small but growing family of three-dimensional PIC simulations of relativistic reconnection with a guide field. With the intention to complement existing work, we initialize our simulations slightly differently than it is normally done, without assuming the usual Harris sheet equilibrium. Also, we explore a parameter regime, involving magnetic-to-kinetic pressure ratios of the order of unity, that has thus far not received sufficient attention. We observe an evolution of magnetic field geometry that constrains the viability of models for high-Lundquistnumber reconnection layers in which the diffusion region contains a hierarchy of interacting plasmoids (e.g., Shibata \& Tanuma 2001; Uzdensky et al. 2010). The simulations also allow us to explore the character of particle energization in dynamical, fully three-dimensional reconnection layers.

The paper is organized as follows. Section 2 describes our methodology and simulation setup, while Section 3 presents the results. Section 4 discusses our findings concerning the development of kinetic instabilities in the current sheet, as well as our findings on particle energization, in view of the existing work on these topics. Finally, Section 5 reviews our main conclusions.

\section{DESCRIPTION OF SIMULATIONS}

\subsection{The Initial Configuration}

The spatial domain is rectangular, with $0 \leqslant x<L_{x}$, $0 \leqslant y<L_{y}$, and $0 \leqslant z<L_{z}$. The boundary conditions are periodic in all directions. The initial magnetic field is the same as that in the Harris equilibrium

$$
\begin{aligned}
\mathbf{B}= & B_{0}\left[\tanh \left(\frac{x-L_{x} / 4}{\lambda_{0}}\right)-\tanh \left(\frac{x-3 L_{x} / 4}{\lambda_{0}}\right)-1\right] \hat{\mathbf{z}} \\
& +\kappa B_{0}(-\hat{\mathbf{y}}),
\end{aligned}
$$

where $\lambda_{0}$ is the half-width of the initial current sheet and $\kappa \geqslant 0$ is a parameter defining the strength of the uniform guide field perpendicular to the opposing field, which in our simulations is oriented in the $-y$ direction. The current sheets are located at $x=L_{x} / 4$ and $x=3 L_{x} / 4$ and carry antiparallel currents. The current density profile that satisfies Ampère's law is

$$
\mathbf{J}=-\frac{c B_{0}}{4 \pi \lambda_{0}}\left[\operatorname{sech}^{2}\left(\frac{x-L_{x} / 4}{\lambda_{0}}\right)-\operatorname{sech}^{2}\left(\frac{x-3 L_{x} / 4}{\lambda_{0}}\right)\right] \hat{\mathbf{y}}
$$

To ensure that Ampère's law is satisfied, if we set the particle density to be uniform, the particles have a spatially-dependent drift velocity $\boldsymbol{\beta}_{i}=-\boldsymbol{\beta}_{e}=\boldsymbol{\beta}$ in the $-y$-direction. The current density $\mathbf{J}$ is related to the velocity $\boldsymbol{\beta}$ by the relation

$$
\mathbf{J}=n_{0} e c\left(\boldsymbol{\beta}_{i}-\boldsymbol{\beta}_{e}\right)=2 n_{0} e c \boldsymbol{\beta},
$$

where $e$ is the magnitude of the unit charge carried by electron and ion macroparticles. This results in the drift velocity profile

$$
\boldsymbol{\beta}=-\beta_{0}\left[\operatorname{sech}^{2}\left(\frac{x-L_{x} / 4}{\lambda_{0}}\right)-\operatorname{sech}^{2}\left(\frac{x-3 L_{x} / 4}{\lambda_{0}}\right)\right] \hat{\mathbf{y}},
$$

where $\beta_{0} \equiv B_{0} /\left(8 \pi n_{0} e \lambda_{0}\right)$.

In this work, we initialize the simulation outside of pressure equilibrium with a uniform initial density $n_{0}$ and spatially varying drift velocity $\boldsymbol{\beta}$. We can still attempt to relate the parameters 
Table 1

Simulation Parameters and Results

\begin{tabular}{|c|c|c|c|c|c|c|c|c|c|c|c|}
\hline Run & $L_{x}{ }^{\mathrm{a}}$ & $L_{y}{ }^{\mathrm{a}}$ & $L_{z}^{\mathrm{a}}$ & $\lambda_{0}{ }^{\mathrm{a}}$ & $\sigma$ & $\kappa$ & $f_{\mathrm{c}}^{\mathrm{b}}$ & $\left|\Delta \mathcal{E}_{B}\right| / \mathcal{E}_{B}(\%)^{\mathrm{c}}$ & $K_{\text {ener }} / K(\%)^{\mathrm{d}}$ & $K_{\text {ener }} /\left|\Delta \mathcal{E}_{B}\right|(\%)^{\mathrm{e}}$ & $\gamma_{\max }^{f}$ \\
\hline S1K0 & 64 & 40 & 40 & 2 & 1 & 0 & 1.5 & 72 & 3.1 & 11 & 48.4 \\
\hline S1K025 & 64 & 40 & 40 & 2 & 1 & 0.25 & 1.0 & 28 & 3.1 & 23 & 34.3 \\
\hline $\mathrm{S} 1 \mathrm{~K} 1$ & 64 & 40 & 40 & 2 & 1 & 1 & 1.0 & 4.3 & 1.5 & 32 & 34.2 \\
\hline $\mathrm{S} 2 \mathrm{KO}$ & 64 & 60 & 60 & 3 & 2 & 0 & 2.3 & 55 & 10 & 30 & 52.6 \\
\hline $\mathrm{S} 2 \mathrm{~K} 025$ & 64 & 60 & 60 & 3 & 2 & 0.25 & 2.3 & 44 & 9.3 & 29 & 54.4 \\
\hline S2K025L & 128 & 120 & 120 & 3 & 2 & 0.25 & 2.3 & 27 & 9.1 & 38 & 58.9 \\
\hline $\mathrm{S} 2 \mathrm{~K} 1$ & 64 & 60 & 60 & 3 & 2 & 1 & 2.3 & 6.7 & 3.2 & 28 & 42.8 \\
\hline
\end{tabular}

Notes.

a The length scales $L_{x}, L_{y}, L_{z}$, and $\lambda_{0}$ are given in units of the plasma skin depth $\lambda_{\mathrm{p}}$.

${ }^{\mathrm{b}} f_{\mathrm{c}}$ is the ratio of the original current sheet width to the current sheet width after the readjustment phase.

$\mathrm{c}$ The ratio $\left|\Delta \mathcal{E}_{B}\right| / \mathcal{E}_{B}$ is the fraction of magnetic energy converted into kinetic energy during the simulation.

${ }^{\mathrm{d}} K_{\text {ener }} / K$ is the particle kinetic energy fraction in energized particles (see Section 3.7.1).

e $K_{\text {ener }} /\left|\Delta \mathcal{E}_{B}\right|$ is the ratio of the particle kinetic energy in energized particles to converted magnetic energy (see Section 3.7.1).

${ }^{\mathrm{f}} \gamma_{\max }$ is the Lorentz factor of the highest-energy particle in the simulation.

of our simulation to those of preceding investigations. In setting up initial conditions for a plasma with the magnetic field given in Equation (1), there are multiple ways in which pressure equilibrium can be satisfied depending on the spatial variation of the plasma density $n_{e^{+}}+n_{e^{-}}$, temperature $T$, charge drift velocity $\boldsymbol{\beta}$, reversing field strength $B_{0}$, and guide field strength $B_{y}$. It is common to assume that the initial temperature is uniform and equal to $T_{0}$, and only the density varies across the current sheet. In practice, Harris sheets are often set up with a strong excess density in the current sheet for pressure balance. It is common to split the particle population into two components, one a uniform background with density $n_{\mathrm{b}}$, and another spatially varying with maximum density $n_{0}$ at the center of the current sheet. The latter component ensures pressure equilibrium and carries the current in the reconnection layer. In Section 2.4 below, we discuss how the initial configuration adjusts to approximate pressure equilibrium.

One can define the magnetic-to-kinetic pressure ratio via

$$
\sigma \equiv \frac{P_{\mathrm{mag}}}{P_{\mathrm{kin}}}=\frac{B^{2}}{8 \pi\left(n_{e^{+}}+n_{e^{-}}\right) T},
$$

where here and henceforth we express the temperature in energy units. Then, in the isothermal Harris sheet pressure equilibria used in preceding investigations, the ratio simply equals the density contrast in the current sheet, $\sigma=n_{0} / n_{\mathrm{b}}$. In our simulation, $\sigma$ is uniform outside the current sheet and equals

$$
\sigma=\frac{B_{0}^{2}}{16 \pi n_{0} T_{0}} .
$$

Note that $\sigma$ is defined not taking into account the magnetic pressure of the guide field.

We do not introduce any initial perturbation to the initial field geometry described here. The structure that develops is thus seeded by numerical fluctuations in conjunction with the initial pressure imbalance.

\subsection{Parameters}

We initialize the simulation at temperature $T_{0}=m_{e} c^{2}$. All the particles are drawn from the relativistic Maxwellian distribution, implying that the average kinetic energy of the particles is $\sim 2.37 m_{e} c^{2}$. We are interested in the dependence of reconnection mechanics and the evolution of particle energy distribution on the dimensionless ratio of the magnetic pressure to the particle pressure $\sigma$, and the guide field amplitude parameter $\kappa$. A nonvanishing $\kappa$ indicates that the magnetic field is twisted in the current sheet and the plasma in the sheet center is magnetized. We run a grid of nine simulations, with $0.25 \leqslant \sigma \leqslant 2$ and $0 \leqslant \kappa \leqslant 1$, and carry out a detailed study of the run with $\sigma=2$ and $\kappa=0.25$. The chosen values of $\sigma$ are low because we are interested in finding the lower limit of magnetization at which reconnection can produce significant particle energization.

Because of the low growth rate, the simulations with $\sigma=0.25$ did not develop the tearing instability over the time period of the simulations, and thus did not undergo reconnection, therefore, we do not show the results of these simulations in what follows. The parameters of the simulations that did undergo reconnection are shown in Table 1.

\subsection{Simulation Method and Resolution Requirements}

We use the relativistic PIC plasma code TRISTAN-MP (Spitkovsky 2008) to simulate the evolution of a reconnection configuration in a pair plasma. In a PIC simulation, the number of macroparticles of each species located in each grid cell must be large enough to resolve variations in the current density and limit high-frequency particle noise. TRISTAN-MP uses a current filtering algorithm to reduce high-frequency particle noise, substantially reducing the required number of macroparticles per cell per species. We initialize our simulations with 4 macroparticles per cell per species, which is fewer than found in other recent PIC simulations (Daughton et al. 2011; Liu et al. 2011). To verify that this low particle density does not lead to cell evacuation, we calculate the total particle density including both species in each cell in Run S2K025 during the flux rope merging phase discussed in Section 3 below; this is the run and time at which the smallest densities are present. We find that the total particle density in each cell calculated with a cubic cloud-in-cell kernel of size equal to the grid spacing is almost always larger than 1 , with a total density larger than 5 in over $93 \%$ of cells. Cells with low particle density occur in regions characterized by weak spatial field gradients that should not be sensitive to particle noise. The cells with strong field gradients that would be sensitive to particle noise invariably have a higher than average particle density. 
To ensure that the particle density is indeed sufficient to resolve the physics of reconnection, we have carried out twodimensional convergence tests, as well as a longer threedimensional test run with 80 particles per cell per species. In our simulations, the tearing instability leading to reconnection is seeded by numerical fluctuations that vary from simulation to simulation, so we do not expect all observables to be the same in each run at a given absolute time measured from the beginning of the simulation. We therefore compare measurable quantities in each simulation at similar points in their evolution; typically, we calculate these quantities at the end of the flux rope merging phase, which is discussed in Section 3 below. The coarse time discretization employed in recording simulation output makes the determination of these evolution points imprecise. This results in inaccuracies in the estimation of observables that vary quickly with time; such quantities will therefore vary with particle density even if the resolution in particle density is sufficient to capture the physics of reconnection.

Our two-dimensional convergence tests use initial conditions matching those in the three-dimensional simulations with $\sigma=2$ and $\kappa=0$, but with a larger number, up to 16 , of macroparticles per species per cell. We find that the only significant discrepancy, up to $40 \%$, is seen in the peak linear growth rate of the kinetic tearing instability; this quantity is very sensitive to the coarse time discretization because time differencing is required for growth rate calculation. This discrepancy is reduced to $25 \%$ in simulations with somewhat finer time discretization in recording outputs; therefore, insufficient particle density is not responsible for the discrepancy in tearing mode growth rates. To test that we have sufficient macroparticle density to account for threedimensional effects in our simulations, we also carry out a three-dimensional simulation with 80 macroparticles per cell per species, and the same parameters as in Run S2K025 with $\sigma=2$ and $\kappa=0.25$. We again find that the measurable quantities do not deviate greatly from those found in Run S2K025, with the maximum deviation of $30 \%$ again seen in the growth rate of the kinetic tearing instability. We also find that the evolution of the total magnetic energy and the particle energy spectrum in this simulation with higher macroparticle density does not differ greatly from that found in Run S2K025 in Section 3.7. The main difference is that in the high-density simulation, the flux rope merging phase identified in Section 3 begins slightly later; this may be understood as the result of slightly lower particle noise in the high density simulations, which results in later growth of instability.

To ensure that our simulations resolve the plasma skin depth, we set $\Delta x=\lambda_{\mathrm{p}} / 8$, where the skin depth is given by

$$
\lambda_{\mathrm{p}}=\sqrt{\frac{\langle\gamma\rangle m_{e} c^{2}}{8 \pi n_{0} e^{2}}} .
$$

Here, $\langle\gamma\rangle$ is the average Lorentz factor of particles in the simulation and the additional factor of two in the denominator reflects the fact that the electrons and positrons oscillate together. To determine whether this value for $\lambda_{p}$ is sufficient to resolve the physics of reconnection, we have carried out further twodimensional convergence test simulations with up to three times larger number of grid cells per skin depth than in the threedimensional simulations, and again compared results at the end of the flux rope merging phase. Here, the most resolutiondependent quantity was the maximum value of the reconnected magnetic field $B_{x}$, which varied by $25 \%$ with resolution. This is a quantity that varies quickly with time near the end of the flux rope merging phase, so the apparent variation with resolution is again likely a result of coarse time discretization.

We focus on the regime in which the particle drift velocity in Equation (4) giving rise to the current density in Equation (2) is nonrelativistic. This places a constraint on the initial current sheet width $\lambda_{0}$. Combining Equations (2) and (3) with the definition of $\sigma$ in Equation (6), it can be shown that

$$
\frac{\lambda_{0}}{\lambda_{\mathrm{p}}}=\frac{\sqrt{2 \sigma(\Gamma-1)}}{\beta_{0}},
$$

where $\Gamma-1 \equiv T_{0} /\left(\langle\gamma\rangle m_{e} c^{2}\right)$ is the ratio of the particle pressure to the particle energy density (which includes the particle rest energy); in our simulations, $\Gamma-1 \approx 0.297 .{ }^{3}$ With this estimate of the relation between $\lambda_{0}$ and $\beta_{0}$, in simulations with $\sigma \leqslant 1$ we choose $\lambda_{0} / \lambda_{\mathrm{p}}=2$, while in simulations with $\sigma=2$ we choose $\lambda_{0} / \lambda_{\mathrm{p}}=3$. This implies that $\beta_{0} \approx 0.36$, ensuring that the drift velocities are not relativistic.

\subsection{Readjustment to Equilibrium}

The uniform initial density and temperature and nonuniform magnetic field in the initial configuration defined by Equations (1), (3), and (4) are not in force balance, but they achieve approximate force balance following a brief readjustment. The initial adjustment to equilibrium results in a reduction of the current sheet thickness and an accompanying compression of the plasma by a factor

$$
f_{\mathrm{c}} \equiv \frac{\lambda_{0}}{\lambda}>1,
$$

where henceforth, $\lambda$ denotes the current sheet half-thickness after the readjustment. We further define

$$
\tau_{c 0} \equiv \frac{\lambda_{0}}{c}, \quad \tau_{c} \equiv \frac{\lambda}{c}
$$

to denote the light crossing times of the initial and the compressed current sheet.

The plasma takes $\approx 10 \tau_{c 0}$ to adjust to a state close to pressure equilibrium. The magnitude of the compression is shown in Table 1 . With readjustment, both the density of the particles and the strength of the guide field increase in the center of the current sheet in such a way as to balance the large pressure gradient that the reversing field exhibits from the center to the surface of the current sheet. Longer-term transients are present in the form of acoustic oscillations; we show that these are magnetosonic waves in Section 3.1. These oscillations could have the effect of enhancing the tearing instability, which relies on a velocity inflow to the current sheet. The initial readjustment does not give rise to any significant magnetic dissipation or particle energization.

\subsection{Unstable Modes and the Size of the Simulation}

For a simulation to capture the physics of reconnection, its size must be sufficient to include the fastest growing modes of the important instabilities of relativistic Harris current sheets

\footnotetext{
3 The index $\Gamma$ is related to the temperature via

$$
\frac{1}{\Gamma-1}=\frac{1}{\theta}\left[\frac{K_{3}\left(\theta^{-1}\right)}{K_{2}\left(\theta^{-1}\right)}-\theta\right]
$$

where $\theta \equiv T /\left(m_{e} c^{2}\right)$ and $K$ is the modified Bessel function of the second kind.
} 
in pair plasmas. The known instabilities include the kinetic relativistic tearing instability (KTI; hereafter the tearing mode), the drift-kink instability (DKI; hereafter the kink mode), and the oblique mode which is similar to the tearing mode. To estimate their wavelengths, we follow Zenitani \& Hoshino (2007). The growth rate $\omega_{\mathrm{KTI}}$ of the tearing mode with wave number $k_{z}$ in a Harris current sheet of half-thickness $\lambda$ and non-relativistic drift velocity $\beta$ is given by

$$
\omega_{\mathrm{KTI}}=b(T) \beta^{3 / 2} k_{z} \lambda\left[1-\left(k_{z} \lambda\right)^{2}\right] \frac{1}{\tau_{c}},
$$

where $b(T)$ is a dimensionless function of the plasma temperature that in the limits of a cold and a relativistically hot plasma equals

$$
b(T)= \begin{cases}\frac{1}{\sqrt{\pi}}\left(\frac{2 T}{\gamma_{\beta} m_{e} c^{2}}\right)^{-1 / 2}, & T \ll m_{e} c^{2}, \\ \frac{2 \sqrt{2}}{\pi}, & T \gg m_{e} c^{2},\end{cases}
$$

and $\gamma_{\beta} \equiv\left(1-\beta^{2}\right)^{-1 / 2}$. The resulting maximum growth rate occurs for $k_{z} \lambda=1 / \sqrt{3}$, corresponding to a wavelength of $\sim 10.8 \lambda$. If we use the form of $b(T)$ appropriate at ultrarelativistic temperatures, then the growth rate for this mode is

$$
\omega_{\mathrm{KTI}, \max }=0.35 \frac{\beta^{3 / 2}}{\tau_{c}} .
$$

The situation is more complicated for the kink mode, because as Zenitani \& Hoshino (2007) find, the analytical maximum value of the growth rate $\omega_{\mathrm{DKI}}$ occurs for $k_{y} \lambda>1$, a wavenumber for which kinetic effects become important in a thin current sheet. Simulation results in their Figure 20 indicate that the maximum growth rate occurs at $k_{y} \lambda \approx 0.7$, corresponding to a wavelength of $\approx 9 \lambda$.

In addition to these two-dimensional modes, it is also necessary to resolve oblique modes with $k_{y}, k_{z} \neq 0$ that combine tearing and kink components; these were identified in threedimensional simulations by Zenitani \& Hoshino (2005, 2008) and Daughton et al. (2011). The typical fastest-growing oblique mode in both of these simulations had $k \lambda \sim 0.2$; this corresponds to a wavelength in both directions of $\sim 30 \lambda$.

To resolve all three types of modes, in all simulations except for the larger size run S2K025L, we set $L_{y}=L_{z}=20 \lambda_{0}$, large enough to contain at least one wavelength of the tearing mode and two wavelengths of the kink mode. Because the initial adjustment leads to a significant narrowing of the current sheet, several wavelengths of the fastest-growing tearing and driftkink modes of the narrower current sheet are included in the simulations, and at least one wavelength of the oblique modes should also be resolved. The resulting overall length scales are $L_{x}=64 \lambda_{\mathrm{p}}$ and $L_{y}=L_{z}=40 \lambda_{\mathrm{p}}$ or $L_{y}=L_{z}=60 \lambda_{\mathrm{p}}$, depending on the simulation, as is shown in Table 1. To ensure that little interaction takes place between the two current sheets in the periodic box, we set $L_{x}=64 \lambda_{\mathrm{p}}$. The current sheets in our larger size simulation $\mathrm{S} 2 \mathrm{~K} 025 \mathrm{~L}$ are as thick as in the other ones, but their separation and the dimensions of the box are twice as large, i.e., $L_{x}=128 \lambda_{\mathrm{p}}$ and $L_{y}=L_{z}=120 \lambda_{\mathrm{p}}$. This simulation was carried out on a $1024 \times 960 \times 960$ grid and contained a total of $\approx 7.5 \times 10^{9}$ particles. The smaller simulations with $\sigma=1$ were carried out on a $512 \times 320 \times 320$ grid and contained $4.2 \times 10^{8}$ particles, whereas the simulations with $\sigma=2$ were carried out on a $512 \times 480 \times 480$ grid with $9.4 \times 10^{8}$ particles.

\subsection{Duration of the Simulations}

In our simulations, the growth times of the tearing and kink modes are typically similar to or smaller than the Alfvén crossing time of the box. To capture the physics of reconnection in its nonlinear regime and on length scales similar to the box size, the duration of the simulation must be larger than these time scales. The relativistic Alfvén velocity is given by

$$
v_{\mathrm{A}}=\frac{c}{\sqrt{1+\Gamma /[2 \sigma(\Gamma-1)]}} .
$$

The Alfvén crossing time of the box $\tau_{\mathrm{A}, z}=L_{z} / v_{\mathrm{A}}$ is $\sim 28 \tau_{c 0}$ in the runs with $L_{z}=60 \lambda_{\mathrm{p}}$. We run all of our simulations for at least 8000 time steps, which amounts to $150 \tau_{c 0}$ in the large simulation S2K025L.

\section{RESULTS}

The evolution of the reconnection layer in all simulations exhibits the same common properties that have been observed in previous PIC simulations of magnetic reconnection in pair plasmas. The tearing instability grows and produces a chain of alternating X-lines and flux ropes. Here, we adopt "flux ropes" to denote a magnetic structure sometimes referred to as "plasmoid" (or "island" in two-dimensional treatments) in which plasma is pinched by a helical magnetic field which can have a braided structure. The flux ropes can have a finite length, with the field lines opening up and extending arbitrarily far from the rope axis. The flux ropes can also split into sub-ropes, which opens the possibility of an organization of the ropes in a flux rope network. In all simulations, except for S1KO where the kink instability disrupts the current sheet, the flux ropes merge in quasi-hierarchical fashion until either only one flux rope is left in each current sheet or, in the large simulation S2K025L, the flux ropes have been disrupted by a transition to a disordered, three-dimensional state. The period of flux rope merging is accompanied by fast magnetic-to-kinetic energy conversion. This conversion produces a tail of energized particles.

In simulations without a guide field, the kink instability also grows, resulting in some corrugation of the current sheet. For $\sigma=2$ in simulation $\mathrm{S} 2 \mathrm{KO}$, the kink instability does not disrupt the quasi-hierarchical merging process. For $\sigma=1$ in simulation S1K0, however, the kinking disrupts the merging and brings the two current sheets into contact where they can interact; this is accompanied by rapid conversion of magnetic to thermal energy but the resulting spectrum does exhibit signatures of a secondary energized component. In agreement with Zenitani \& Hoshino (2008), we find that the presence of a guide field suppresses the kink instability. However, we find that in the large simulation S2K025L, a transition to three-dimensional evolution still occurs, likely due to a lack of large-scale phase coherence in the tearing instability.

We proceed to discuss our results in detail. In Section 3.1, we discuss the evolution of the global current sheet and the formation of what will turn out to be a network of interconnected flux ropes in our largest simulation. In Section 3.2, we identify sites of magnetic reconnection within the network. In Section 3.3, we carry out a Fourier decomposition of perturbations in the largest simulation and discuss their growth rates. In Section 3.4, we analyze the time scales associated with the evolution of the flux rope network. In Section 3.5, we measure the overall reconnection rate, while in Section 3.6 we analyze components of the nonideal electric field as reflected in 

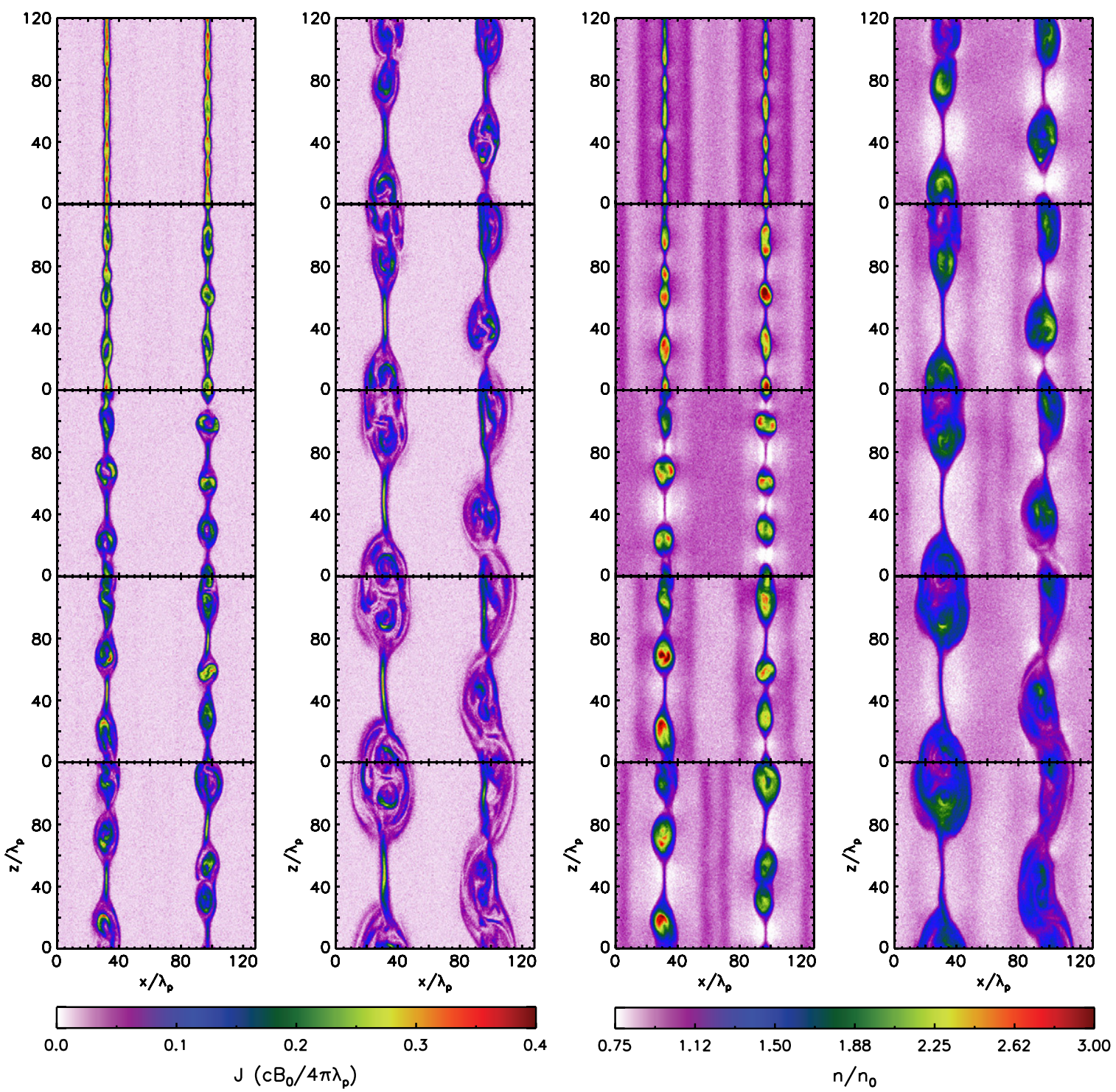

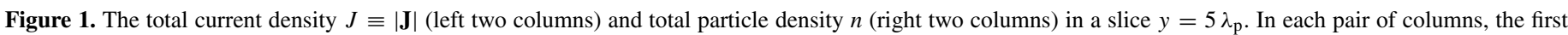

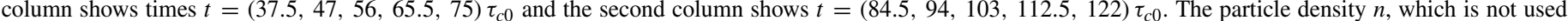

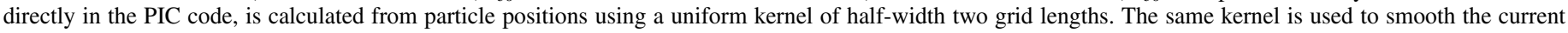
density $J$.

(A color version of this figure is available in the online journal.)

the generalized Ohm's law. Finally, in Section 3.7, we discuss the overall rate of magnetic-to-thermal energy conversion as well as the efficiencies, mechanisms, and properties of particle energization in the simulations.

\subsection{Formation and Evolution of the Flux Rope Network}

Figure 1 shows a time sequence of slices at $y=5 \lambda_{\mathrm{p}}$ of the total current density $J \equiv|\mathbf{J}|$ and the plasma number density $n$ in simulation S2K025L. The early evolution of each current sheet, if observed at a single value of $y$, is that correctly described by the familiar flux rope (or island, plasmoid) merging paradigm. Larger flux ropes produced by the merging of smaller ones contain substructure reflected in multiple, curved, embedded current sheets, each with a half-thickness $\sim \lambda_{\mathrm{p}}$. Very similar substructure also appears in the recent two-dimensional PIC simulations of an electron-ion plasma by Markidis et al. (2012).
Outside of the two evolving current sheets, plane-parallel magnetosonic waves resulting from the initial pressure imbalance can be seen in the plasma density, the electric field in the $y$ direction, and the magnetic field in the $z$ direction. The waves traverse the computational box several times in the course of the simulation, moving with a typical speed of approximately $0.8 c$. To check that these are indeed magnetosonic waves, we calculate the magnetosonic speed $v_{\mathrm{ms}}=c \sqrt{\left(v_{\mathrm{A}}^{2}+c_{\mathrm{s}}^{2}\right) /\left(v_{\mathrm{A}}^{2}+c^{2}\right)}$, where $c_{\mathrm{s}}$ is the local sound speed given by $c_{\mathrm{s}}=c \sqrt{\Gamma-1} \sim 0.55 c$. Because the typical density in the background plasma is $\sim 0.8 n_{0}$ and a guide field of magnitude one quarter that of the reversing field is present, the Alfvén speed in the background plasma is approximately $0.73 c$. The magnetosonic speed in the background plasma is therefore approximately $0.74 c$, which is similar to the speed of the observed waves.

To examine three-dimensional aspects of the current sheet evolution, in Figure 2 we show projections into the $y z$ plane of 

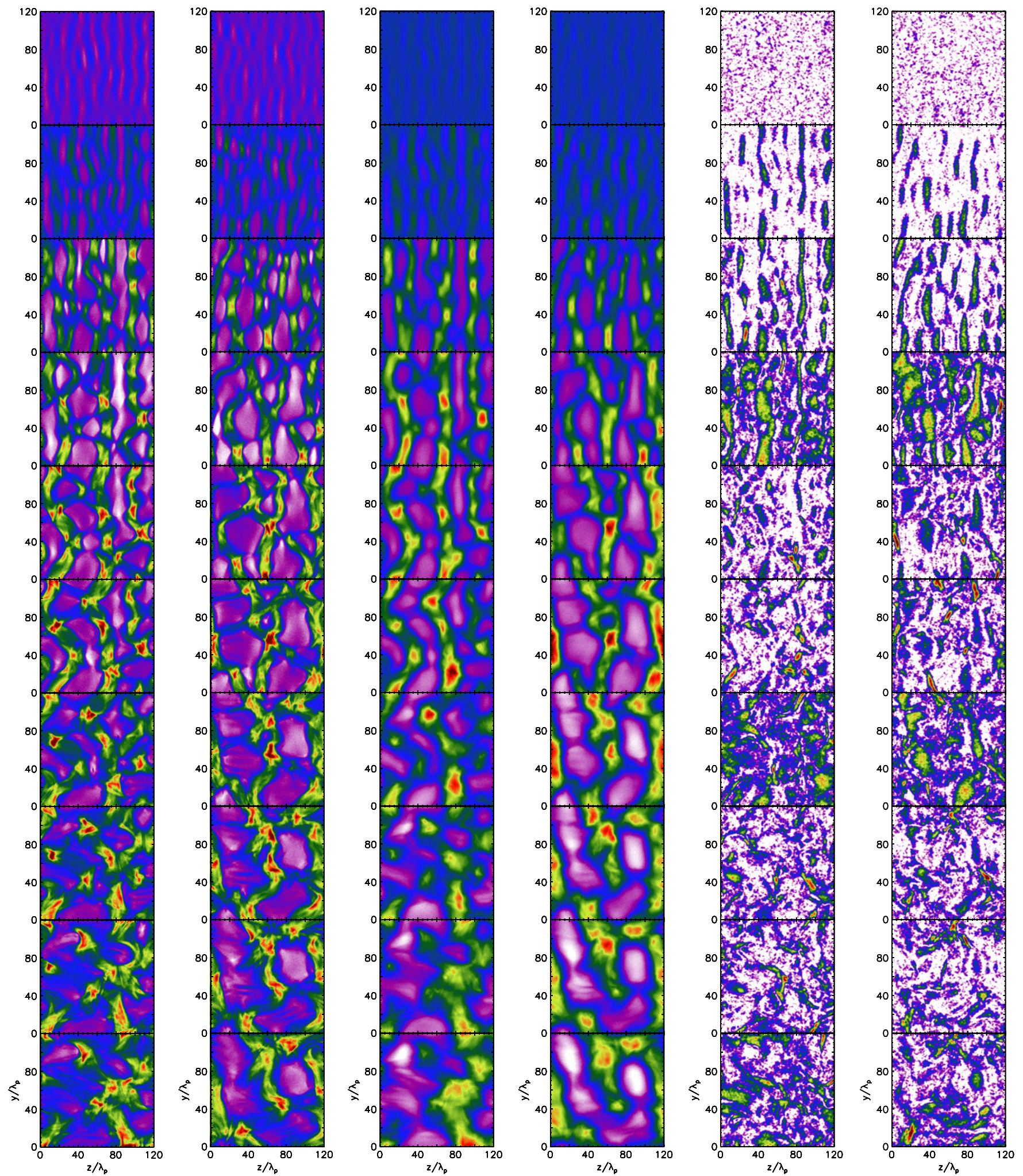

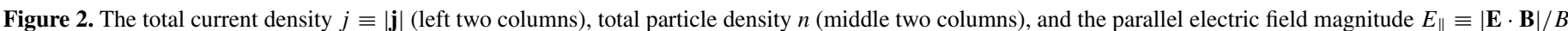

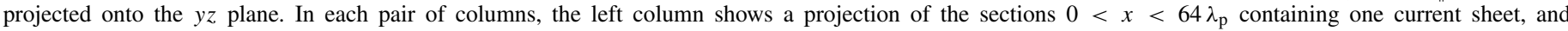

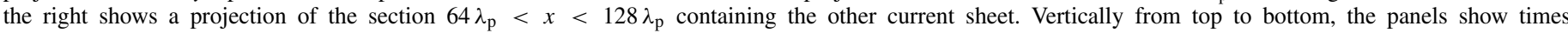

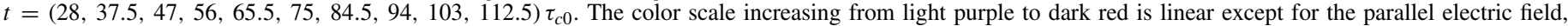

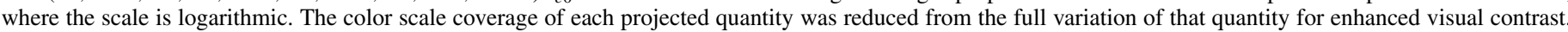

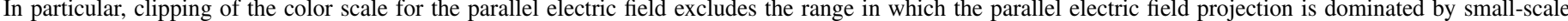

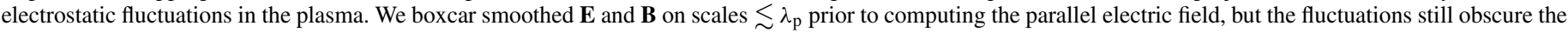
variation of parallel field in the current sheet at the earliest time shown, $t=28 \tau_{c 0}$.

(A color version of this figure is available in the online journal.) 
the total current density $J$, plasma number density $n$, and the parallel electric field magnitude $E_{\|}$in each of the two current sheets in simulation S2K025L. The flux ropes that initially develop from the linear tearing instability are approximately parallel to the $y$ axis, which is the direction of the initial current flow. However, each flux rope exhibits one or more discontinuities where the $z$ coordinate of the rope suddenly changes. We interpret these discontinuities as arising from a lack of tearing mode phase coherence on scales larger than those in causal contact during the initial development of the instability. The lack of phase coherence should occur on scales

$$
\Delta y, \Delta z \gg \lambda_{\text {coh }} \sim \frac{c}{\omega}
$$

on which locations on the current sheet are out of causal contact during the first $e$-folding of the instability; here, $\omega$ is the linear growth rate of a mode growing from numerical noise. This sets a hard upper limit on the scale on which an instability can grow coherently; other constraints may limit this scale further.

The initial departure from perfect translational invariance in the $y$ direction implies that the nonlinear flux rope merging will itself not occur coherently. In any three adjacent flux ropes, the middle rope can merge with one of the flanking ropes at one $y$, and with the other rope at another $y$; at still other values of $y$, the three ropes may remain separate for a time being. This creates lateral linkage between the flux ropes; already at time $t=37.5 \tau_{c 0}$, all the flux ropes are mutually linked inside their current sheet. The linkage implies each flux rope experiences a strongly $y$-dependent magnetic tension force. This magnetic tension from neighboring flux ropes leads to rope tilting and kinking that is very distinct in origin from that arising from the well-known linear oblique and kink instabilities of a current sheet.

Pairs of large flux ropes emerging from two generations of flux rope merging, but still oriented largely parallel to the $y$ axis, are often connected by minor flux ropes that are highly tilted toward the $z$ axis. Daughton et al. (2011) have discovered similar structures in a large three-dimensional simulation of electronion reconnection with a strong guide field. It is clear in Figure 2 that by $t \sim 75 \tau_{c 0}$, all flux rope segments have substantial tilts and any semblance of translational invariance in the direction of the original current flow is lost. Vertices of the flux rope network contain highly localized, intense current sheets and filaments. By $t \sim 122 \tau_{c 0}$, the flux ropes are largely completely disrupted and the current sheet contains a disordered network of knot- and sheet-like structures.

The force $\mathbf{J} \times \mathbf{B}$ peaks at the primary X-line current sheets as well as flux rope perimeters, but is on average much smaller in flux rope interiors. This suggests that the flux rope interiors are organized in a state of force free quasi-equilibrium constrained by a nonvanishing magnetic helicity (i.e., twisting, braiding of the field lines).

\subsection{Dissipation and Reconnection in the Flux Rope Network}

To gain further insight into the structure of the flux rope network, we examine the spatial variation of $\mathbf{E}_{\|}$, the component of the electric field parallel to the magnetic field. The parallel field vanishes for the purely inductive electric field of ideal MHD, and thus it can be obtained purely from the non-ideal, reconnection electric field $\mathbf{R}$ defined as the difference between the actual and the induction electric field,

$$
\mathbf{R} \equiv \mathbf{E}+\frac{1}{c}(\mathbf{v} \times \mathbf{B}) .
$$

In high magnetic Reynolds number plasmas, the parallel field is present only in magnetic diffusion regions, and in particular, in locations where a change of magnetic connectivity is under way (e.g., Schindler et al. 1988). ${ }^{4}$ The parallel electric field may also be instrumental in maintaining the pressure anisotropy, which is responsible for the breaking of flux freezing in the reconnection layer (see, e.g., Hesse et al. 2011).

The two rightmost columns of Figure 2 show that the magnitude of the parallel electric field $E_{\|} \equiv\left|\mathbf{E}_{\|}\right|$strongly peaks in the thin, long current sheets containing primary $\mathrm{X}$-lines and connecting magnetic flux ropes. However, prior to flux rope disruption and the transition to a disordered state, the parallel field is normally very small inside magnetic flux ropes even when they contain embedded, substructure-level current sheets. An exception is flux rope segments directly undergoing dynamical transformation, such as merging or stretching, where very isolated, spatially and temporally intermittent sites of significant $E_{\|}$occasionally appear. The flux rope interiors are not undergoing pervasive, steady reconnection, in spite of their complex magnetic substructure. The intermittent $E_{\|}$ is also common after the flux rope network has become disordered, indicating that the disordered network remains active by developing transient, localized dissipation events in the braided network of disrupted flux ropes that effect further evolution of magnetic connectivity.

Reconnection intermittency is also evident in Figure 3, showing views of the current sheets $t=56 \tau_{c 0}$ during the initial flux rope merging phase, and at $t=84.5 \tau_{c 0}$ after the flux rope network has become disordered. The field lines shown are those passing through the sites where $E_{\|}$is significant, where the field line color is shown in green and field line geometry is that of an $\mathrm{X}$-line. The reconnected magnetic field snaps back to become incorporated into that of the flux ropes. It is also noticeable that the flux ropes are not cylindrically symmetric but highly flattened (ribbon-like) and exhibit a clear longitudinal twist.

A common measure of magnetic connectivity is the concept of magnetic helicity, but its definition on the entire periodic domain presents unique challenges (e.g., Berger 1997 and references therein). In spatially and temporally localized, simplyconnected reconnection regions, a generalized helicity can be meaningfully defined using the Finn-Antonsen approach (e.g., Berger 1999). In guide-field reconnection, a change of the generalized helicity implies a change of global field line connectivity (Schindler et al. 1988). Since the generalized helicity is locally generated by the source term $-2 \mathbf{E} \cdot \mathbf{B}=-2 \mathbf{R} \cdot \mathbf{B}$, which is closely related to the parallel electric field, the twisting and braiding of the field lines interior to a flux rope is a consequence of (possibly $y$-coordinate dependent) field connectivity change in the primary $\mathrm{X}$-line region. ${ }^{5}$

\subsection{Instability Mode Analysis}

To identify modes present in the simulation, we carried out Fourier decomposition of the electric and magnetic field for wave vectors $\mathbf{k}$ parallel to the plane of the current sheet, $k_{x}=0$, in simulation S2K025L. We search for signatures of the tearing and kink instability, as well as for oblique modes, see, e.g., Daughton et al. (2011) and Baalrud et al. (2012). The tearing instability has $\mathbf{k} \| \hat{\mathbf{z}}$ and creates a perturbation in $B_{x}$ and $E_{y}$.

\footnotetext{
4 On very small scales in the simulation, $\mathbf{E}_{\|}$exhibits noise in the entire domain due to small scale electrostatic fluctuations; in our analysis, we filter these fluctuations on scales $\lesssim 2 \lambda_{\mathrm{p}}$.

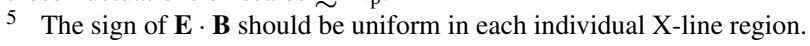



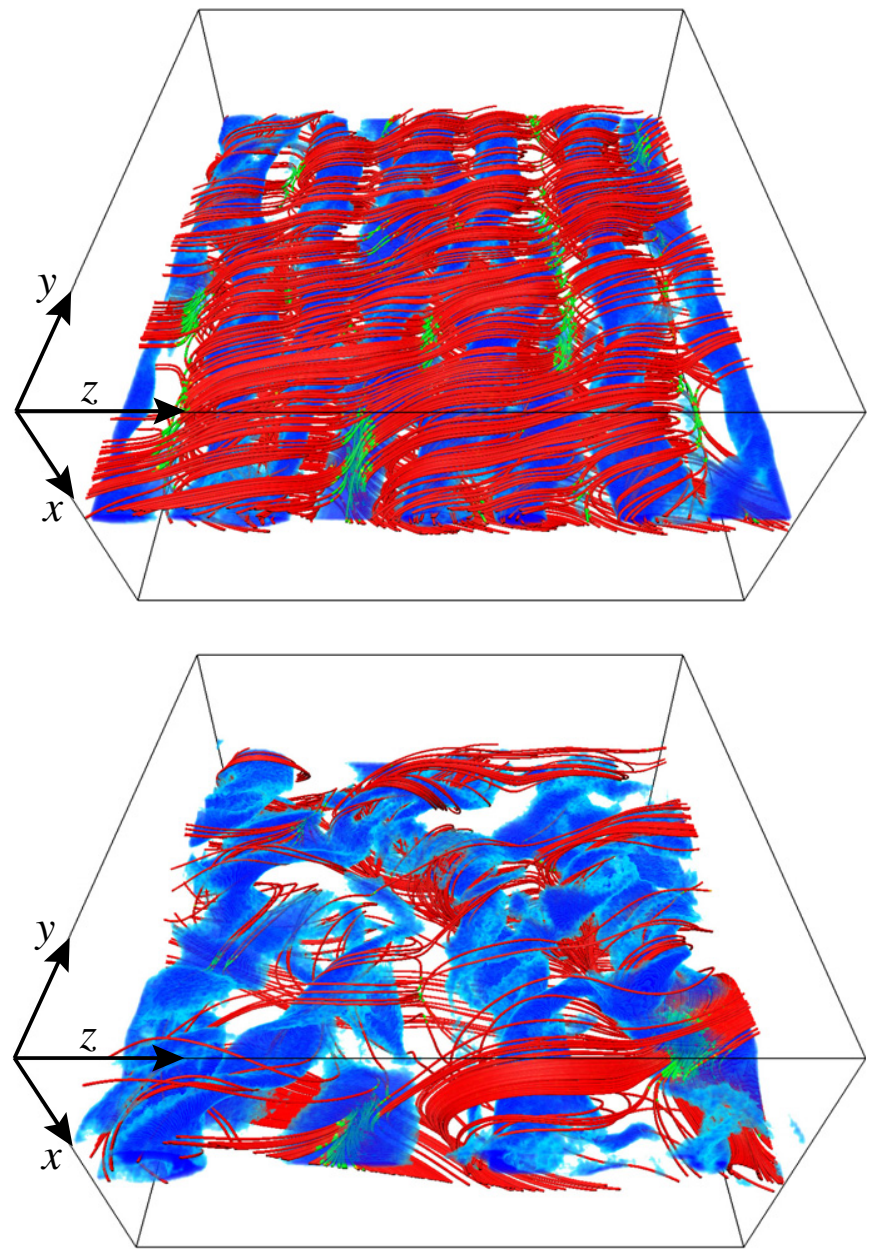

Figure 3. A view of the current sheet at $x=L_{x} / 4$ at times $t=56 \tau_{c 0}$ (upper panel) and $t=84.5 \tau_{c 0}$ (lower panel). The volume rendering (blue color) shows the region with current density $J>0.2 c B_{0} /\left(4 \pi \lambda_{\mathrm{p}}\right)$ (upper panel) and $J>0.12 c B_{0} /\left(4 \pi \lambda_{\mathrm{p}}\right)$ (lower panel). The curves are the magnetic field lines that pass through regions of high parallel electric field $E_{\|}$, and thus are actively undergoing reconnection. The fraction of the field line with an enhanced value of $E_{\|}$is rendered in green.

(A color version of this figure is available in the online journal.)

The kink instability has $\mathbf{k} \| \hat{\mathbf{y}}$ and in the linear order creates a perturbation in $E_{z}$ if a guide field is present. The oblique mode is similar to the tearing mode, except that the wave vector is tilted in the $y z$ plane, that of the initial current sheet. In Figure 4, we show Fourier power spectra of the magnetic field component $B_{x}$ in the linear phase at $t=19 \tau_{c 0}$ and at the end of the fully nonlinear flux rope merging phase at $t=37.5 \tau_{c 0}$. We also show the corresponding growth rates evaluated over an interval of $\Delta t=9 \tau_{c 0}$ preceding each of the two times,

$$
\omega_{B_{x}}(\mathbf{k}, t) \equiv \frac{\ln \left|B_{x}(\mathbf{k}, t) / B_{x}(\mathbf{k}, t-\Delta t)\right|}{\Delta t} .
$$

In the figure, the growth rates are multiplied by the light crossing time of the compressed current sheet $\tau_{c} \approx 0.43 \tau_{c 0}$. The modes with fastest growth rates are clustered around the $k_{z}= \pm 2 \pi\left(L_{z} / 9\right)^{-1}$ tearing mode at $t=19 \tau_{c 0}$, but the fastest growth then shifts to longer wavelengths and oblique directions by $t=37.5 \tau_{c 0}$.

At the end of the linear phase, the power spectrum for $B_{x}$ and $E_{y}$ peaks at $(2 \pi)^{-1}\left(k_{y}, k_{z}\right)=\left(0, \pm\left(L_{z} / 9\right)^{-1}\right)$, which is the tearing mode, consistent with the presence of $\sim 9$ flux
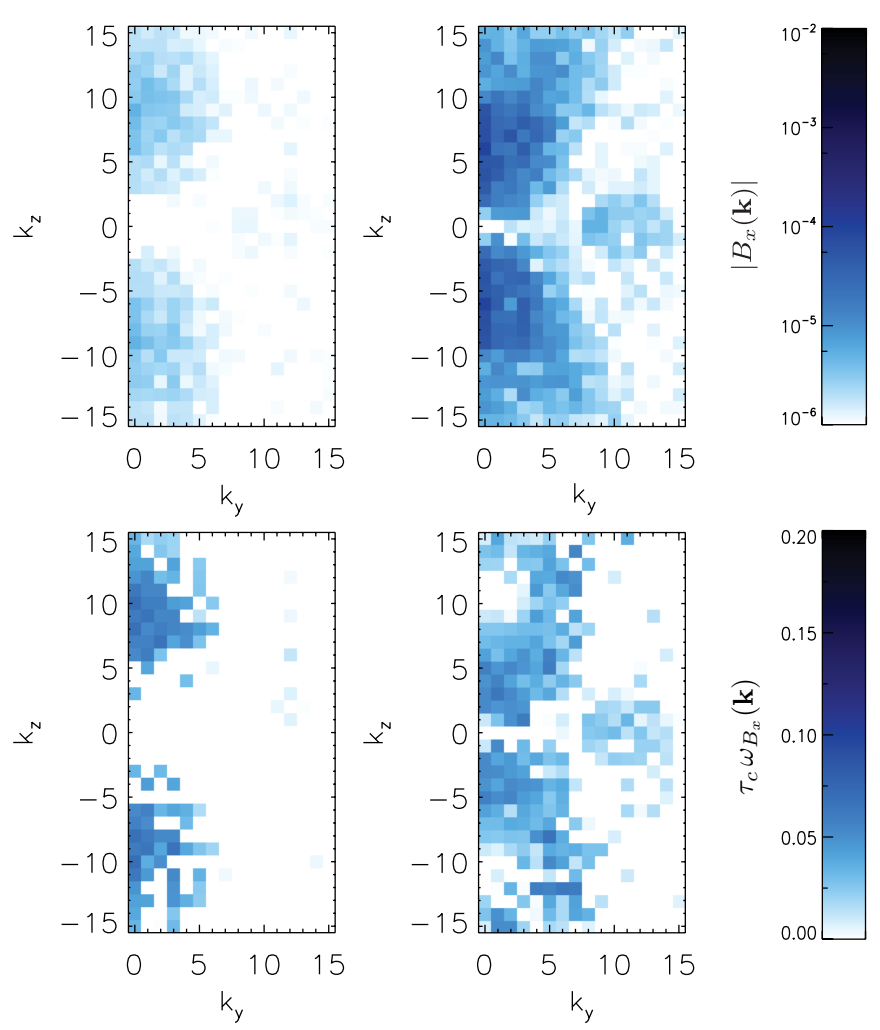

Figure 4. Fourier mode amplitudes (top panels) and growth rates (bottom panels) of $B_{x}$ in one of the current sheets at times $t=19 \tau_{c 0}$ (left panels) and $t=37.5 \tau_{c 0}$ (right panels) in simulation S2K025L. The amplitude was computed with the Fast Fourier Transform via $\mathbf{B}(\mathbf{k})=\left(N_{x} N_{y} N_{z}\right)^{-1} \sum_{i_{x}=1}^{N_{x}} \sum_{i_{y}=1}^{N_{y}}$ $\sum_{i_{z}=1}^{N_{z}} \exp \left[-2 \pi i\left(i_{y} k_{y} / N_{y}+i_{z} k_{z} / N_{z}\right)\right] \mathbf{B}\left(i_{x}, i_{y}, i_{z}\right)$, where $N_{x}, N_{y}$, and $N_{z}$ are dimensions of the computational grid, and the initial reversing field $B_{0}=0.1$ in these units. The components of the wave vector are expressed in units of $2 \pi$ divided by the computational box size along the relevant direction. We only show growth rates for modes with amplitudes $\left|B_{x}(\mathbf{k})\right|>10^{-6}$.

(A color version of this figure is available in the online journal.)

ropes in each current sheet. The peak, however, is broadened, likely by an initial lack of phase coherence on scales given by Equation (16). For the tearing mode, $\omega_{\mathrm{KTI}} \sim 0.083 \tau_{c}^{-1}$. From this we expect a lack of phase coherence on scales $\Delta y, \Delta z \gg \lambda_{\text {coh, KTI }} \sim 5.2 \lambda_{0} \sim 16 \lambda_{\mathrm{p}}$, which can explain the observed broadening. However, the broadening also allows the possibility that an authentic oblique component is present too. At the end of the flux rope merging phase, which we define as the time $t \sim(50-60) \tau_{c 0}$ when the orderly flux rope merging gives way to a more disordered evolution, the peak has shifted to longer wavelengths $\left(L_{z} / 2\right)^{-1} \leqslant(2 \pi)^{-1}\left|k_{z}\right| \leqslant\left(L_{z} / 6\right)^{-1}$ with significant additional power occurring in the oblique direction, for $(2 \pi)^{-1} k_{y} \leqslant\left(L_{y} / 3\right)^{-1}$. We emphasize, again, that the oblique power may be an outcome of phase decoherence, as well as of secondary instabilities developing in the nonlinear regime, rather than of a genuine primary oblique instability mode.

Turning to our search for the kink mode, we find that $E_{z}$ develops nonvanishing albeit weak $\mathbf{k} \| \hat{\mathbf{y}}$ power at $t \gtrsim$ $40 \tau_{c 0}$ with the peak wavelength corresponding to $(2 \pi)^{-1} k_{y} \cong$ $\left(L_{y} / 3\right)^{-1}$. However, most of the power in $E_{z}$ is still along $\mathbf{k} \| \hat{\mathbf{z}}$. Other components of $\mathbf{E}$ and $\mathbf{B}$ are devoid of power along $\mathbf{k} \| \hat{\mathbf{y}}$. This suggests that the simulation exhibits no evidence for a linear kink mode and that any variation with $y$ emerges in the nonlinear development of the tearing mode. We compare these results to previous analyses of tearing, kink, and oblique modes in Section 4.1 below. 


\subsection{Flux Rope Merger Timescales}

Once the tearing instability has produced a collection of nonlinear flux ropes at a time that we denote with $\tau_{\mathrm{NL}}$, the ropes begin to merge. The merging time should scale with the island separation $\Delta z$ as

$$
\tau_{\text {merge }} \sim \frac{\Delta z}{\chi v_{\mathrm{A}}},
$$

where $\chi$ is a dimensionless coefficient encapsulating the dynamics of interaction between flux ropes. Then, the number of flux ropes per unit length along the current sheet, which we denote with $\mu$, can be estimated as

$$
\mu \sim \frac{1}{\chi v_{\mathrm{A}}\left(t-\tau_{\mathrm{NL}}\right)} .
$$

By visually counting the number of flux ropes in each current sheet in simulation S2K025L in Figure 2, we find that

$$
\mu \approx \frac{1.2}{\lambda_{\mathrm{p}}}\left(\frac{t}{\tau_{c 0}}-16\right)^{-1},
$$

suggesting that $\tau_{\mathrm{NL}} \approx 16 \tau_{c 0}$ in that simulation. Comparing Equations (20) and (21), we estimate that $\chi \approx 0.4$. Given that the reconnection outflow velocities reach only about a half of the Alfvén velocity in the simulation, it is not surprising that the characteristic velocity associated with flux rope merging $\chi v_{\mathrm{A}}$ is only a fraction of the Alfvén velocity.

In reconnection configurations starting from a onedimensional current sheet lacking any $y$ and $z$ dependence, the three-dimensionality manifested in an emerging $y$ dependence can arise from several effects which include an intrinsic obliqueness of the tearing modes, the development of kink modes, and a lack of coherence on scales on which the phase of the tearing mode is uncorrelated (see Section 2.5). In reconnection regimes in which oblique and kink modes are suppressed, flux rope dynamics acquires a three-dimensional character when the flux rope separation $\sim \mu^{-1}$ starts substantially exceeding the coherence length $\lambda_{\text {coh,KTI }}$. From Equations (14), (16), and (20), this happens at times

$$
t \gg \tau_{3 \mathrm{D}, \mathrm{coh}} \sim \tau_{\mathrm{NL}}+\frac{\lambda}{0.35 \chi v_{\mathrm{A}} \beta^{3 / 2}},
$$

yielding an estimate $\tau_{3 \mathrm{D} \text {,coh }} \sim 36 \tau_{c 0}$ for simulations S2K025 and S2K025L. Indeed, the flux rope geometry in Figure 2 seems to acquire three-dimensional character at $t \gtrsim(40-50) \tau_{c 0}$. This is consistent with the rise of power at oblique wave vectors $k_{y}>0$ in Figure 4 at similar times. This analysis suggests that the reconnection layers acquire three-dimensional structure on relatively short time scales regardless of the growth rates of the oblique and kink modes.

\subsection{The Reconnection Rate}

We now discuss the reconnection efficiency at primary $\mathrm{X}$-lines in the early stages of the evolution of the current sheet. In this regime, the reconnection site can be described using a typical two-dimensional model of reconnection in which two oppositely oriented fields are separated by a thin current layer in which the field reverses. In this layer, magnetic field lines diffuse across the plasma to reconnect at one or more X-lines. Magnetized plasma approaches the central plane of the layer, toward an X-line, with an asymptotic inflow velocity $v_{\text {in }}$, which is also known as the reconnection velocity. After passing the $\mathrm{X}$-line, the plasma is expelled from the vicinity of the X-line to either side at the outflow velocity $v_{\text {out }}$, which is typically assumed to equal the Alfvén velocity $v_{\mathrm{A}}$. The orientation of the reconnected magnetic field is approximately perpendicular to that of the original field.

Defining $\delta$ and $L$ to be the thickness and length of the current sheet, the conservation of mass from the inflow to the outflow requires

$$
\frac{\delta}{L} \approx \frac{v_{\text {in }}}{v_{\text {out }}} \approx \frac{v_{\text {in }}}{v_{\mathrm{A}}}
$$

The inflow velocity can be related to the electric field in the reconnection region $\mathbf{E}_{R R}$, which is approximately uniform and equal to the dynamical electric field in the plasma inflow just outside the current sheet

$$
\mathbf{E}_{\mathrm{RR}} \approx \frac{1}{c}\left(\mathbf{v}_{\mathrm{in}} \times \mathbf{B}_{0}\right)
$$

Because the inflow velocity and the reversing magnetic field are perpendicular, the dimensionless reconnection rate $r_{\text {rec }}$, defined as the ratio of the inflow and the outflow velocity, and the electric field in the reconnection region, can be related via

$$
r_{\text {rec }} \equiv \frac{v_{\text {in }}}{v_{\text {out }}}=\frac{E_{\mathrm{RR}}}{\left(v_{\mathrm{A}} / c\right) B_{0}} .
$$

This calculation of the reconnection rate makes the common assumption that the outflow velocity is equal to the Alfvén velocity in the inflow region. In our simulations, this assumption is not valid as the outflow velocity is smaller than the Alfvén velocity.

In simulation S2K025, we find that indeed, the total electric field in the reconnection region is approximately uniform across the current sheet. Given that the Alfvén velocity is $v_{\mathrm{A}} \approx 0.7 c$ in this simulation, the dimensionless reconnection rate calculated from the electric field following Equation (25) is $r_{\text {rec }} \approx 0.05$ at $t=37.5 \tau_{c 0}$, in the middle of the initial flux rope merging phase. This is similar to the peak reconnection rates of about $0.07-0.1$ found in other three-dimensional PIC simulations with higher magnetizations (e.g., Zenitani \& Hoshino 2008; Liu et al. 2011). This comparison assumes that reconnection at the largest $\mathrm{X}$-line can be described accurately by a two-dimensional reconnection model. The inflow and outflow velocities at $t=37.5 \tau_{c 0}$ are $v_{\text {in }} \approx 0.02 c$ and $v_{\text {out }} \approx 0.27 c$, respectively. The peak plasma outflow velocity slightly later in the simulation reaches $v_{\max } \approx 0.39 c$, just above half of the Alfvén velocity. Since the Alfvén velocity is not ultrarelativistic, it is not surprising that we are not finding ultrarelativistic flows in any of our simulations. ${ }^{6}$ If the dimensionless reconnection rate is calculated directly from the inflow and outflow velocities, then it is $r_{\text {rec }} \approx 0.08$. The difference between the outflow velocity and the Alfvén velocity, which are equal in idealized two-dimensional reconnection models, is likely due to the dynamical, non-steady state nature of the plasma flow in our simulations; similar discrepancies were found by Bessho \& Bhattacharjee (2010) in their nonrelativistic PIC simulations, and Takahashi et al. (2011) in their relativistic MHD simulations.

\footnotetext{
6 To obtain an ultrarelativistic flow, one must have $\gamma_{\mathrm{A}} \equiv\left[1-\left(v_{\mathrm{A}} / c\right)^{2}\right]^{-1 / 2} \gg 1$, but this may not be sufficient, e.g., Zenitani \& Hoshino (2008) found maximum flow speeds of $v_{\text {out }} \approx(0.7-0.8) c$ even in their two-dimensional simulations with high magnetization and no guide field.
} 


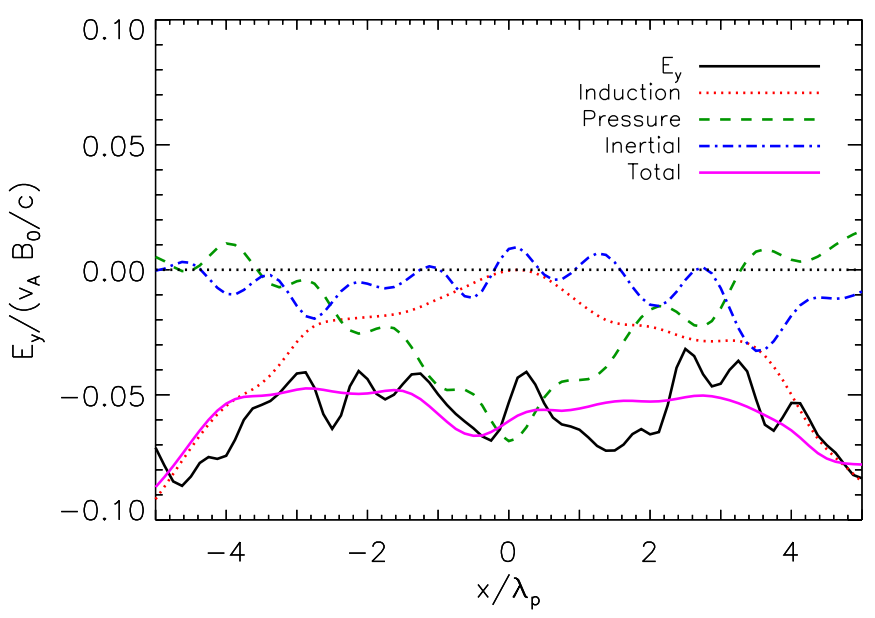

Figure 5. The variation of the terms in the generalized Ohm's law of the electric field across the current sheet near the largest X-line at $t=47 \tau_{c 0}$. The center of the current sheet is at $x=0$. The lines represent the measured electric field $E_{y}$ (solid, black), the induction component (dotted, red), the pressure component (dashed, green), the inertial component (dot-dashed, blue), and the total (pink, solid) of the three calculated electric field terms. The generalized Ohm's law mandates that the latter should equal the actual electric field, which is the case apart from fluctuations arising from numerical discreteness. The Ohm's law components are smoothed with a Gaussian kernel of standard deviation $0.33 \lambda_{\mathrm{p}}$. It is clear that at the $\mathrm{X}$-line, the pressure term dominates over the inertial term.

(A color version of this figure is available in the online journal.)

\subsection{Generalized Ohm's Law Analysis}

A central question in magnetic reconnection is the nature of the process that facilitates the violation of flux freezing in the diffusion region. The total electric field $\mathbf{E}$ in a collisionless plasma is related to the magnetic field $\mathbf{B}$ and to moments of the particle distribution function of particle species $s$ by the generalized Ohm's law (e.g., Krall \& Trivelpiece 1973)

$$
\begin{aligned}
\mathbf{E}+\frac{1}{c}\left\langle\mathbf{v}_{s}\right\rangle \times \mathbf{B}= & \frac{1}{q_{s} n_{s}} \nabla \cdot \mathbf{P}_{s} \\
& +\frac{1}{q_{s}}\left(\frac{\partial\left\langle\mathbf{p}_{s}\right\rangle}{\partial t}+\left\langle\mathbf{v}_{s}\right\rangle \cdot \nabla\left\langle\mathbf{p}_{s}\right\rangle\right),
\end{aligned}
$$

where $\left\langle\mathbf{v}_{s}\right\rangle$ and $\left\langle\mathbf{p}_{s}\right\rangle$ are the average velocities and momenta of particles, $\mathbf{P}_{s}$ is the pressure tensor, $n_{s}$ is the particle number density, and $q_{s}$ and $m_{s}$ are the particle charge and mass. Averaging over the two species $s=\{i, e\}$, we recover the non-ideal, reconnection electric field $\mathbf{R}$ on the left hand side of Equation (26). In collisionless plasmas with long-lived magnetization (counterexamples being the plasmas excited by kinetic instabilities, such as the filamentation instability), the pressure and the inertial terms on the right hand side of Equation (26) are small almost everywhere. Both of these terms, however, can become important in the diffusion region of a magnetic reconnection layer.

To determine which terms give rise to $\mathbf{R}$, we calculate the terms on both sides of Equation (26) as they vary across the $\mathrm{X}$-line in simulation S2K025. We find no significant reconnection electric field in the $x$ or $z$ directions; there is only a reconnection field in the $y$ direction, consistent with two-dimensional models of reconnection. Meanwhile, during the linear growth phase of the tearing instability, spatial gradients are present only in the $x$ and $z$ directions, implying that only the off-diagonal $x y$ and $z y$ components of the pressure tensor contribute to the first term on the right hand side of Equation (26). In particular, we find that most of the pressure term is provided by $\partial P_{x y} / \partial x$.

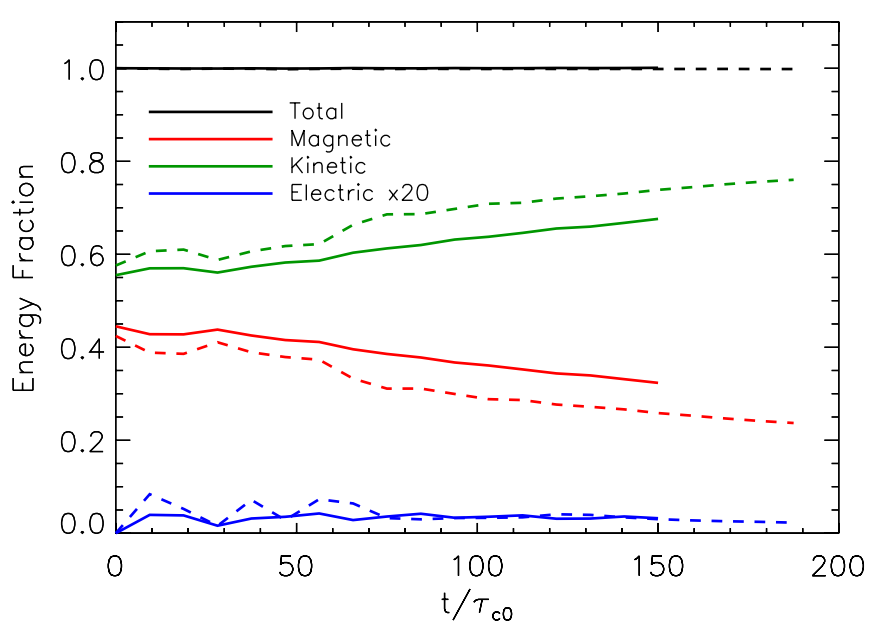

Figure 6. Evolution of the total magnetic energy (red lines), particle kinetic energy (green lines), and the energy in the electric field multiplied by 20 for clarity (blue lines), all normalized to the total initial energy, in runs S2K025L (solid lines) and S2K025 (dashed lines). The time is expressed in units of the light crossing time of the current sheet $\tau_{c 0}$.

(A color version of this figure is available in the online journal.)

Figure 5 shows the $y$ component of various terms contributing to the electric field. The pressure term is dominant, and the total of the inductive, pressure, and inertial terms approximates the electric field, with departures arising from shot noise.

These results are consistent with previous investigations (e.g., Bessho \& Bhattacharjee 2005, 2007; Schmitz \& Grauer 2006) that found that the spatial variation of off-diagonal terms of the pressure tensor was the main contributor to the reconnection electric field. The results of our Ohm's law calculation with a moderate guide field, combined with the similar results of other simulations with strong guide field (Che et al. 2011, nonrelativistic simulations) and no guide field (Liu et al. 2011, relativistic simulations), constitute evidence that for moderate magnetizations and all guide field magnitudes, the reconnection electric field is produced by the pressure term in three as well as in two dimensions. In an exception to this agreement, Hesse \& Zenitani (2007) found that the inertial term became important in highly magnetized relativistic current sheets with a guide field, which is a regime we do not probe.

\subsection{Particle Energization}

We now turn to the topic of magnetic-to-kinetic energy conversion and particle energization. Figure 6 shows the evolution of the total magnetic field, electric field, and particle kinetic energies in simulations S2K025 and S2K025L. Energy was conserved to within $0.3 \%$ in all the simulations. The initial kinetic and electromagnetic energy fraction differs in the two simulations because the initial current sheet occupies a fraction of the volume of the simulation box that is twice as large in simulation S2K025 as in S2K025L. The trends, however, are consistent when taking the volume difference into account. An initial reversible fluctuation of the magnetic-to-kinetic ratio during the first $\sim 20 \tau_{c 0}$ is associated with the readjustment to pressure equilibrium. Dissipative conversion of magnetic to kinetic energy seems to first occur in a couple of bursts at $t \sim(30-40) \tau_{c 0}$ and $t \sim(50-60) \tau_{c 0}$. Then, the system enters a phase, lasting until the end of the simulation, in which dissipation is relatively steady. The two bursts seem to coincide with initial tearing instability and the formation of $\sim 9$ flux ropes (magnetic islands) across the simulation box per current sheet, and the subsequent 
flux rope merging, yielding $\sim 3$ flux ropes per current sheet by $t \sim 60 \tau_{c 0}$, after which the overall geometry of the reconnection layer becomes fully three dimensional. By $t=150 \tau_{c 0}$, about $40 \%$ and $20 \%$ of the initial magnetic energy is converted into particle kinetic energy in the smaller and larger simulation, respectively.

In Table 1 , we show $\left|\Delta \mathcal{E}_{B}\right| / \mathcal{E}_{B}$, the fraction of magnetic field energy that is converted to particle kinetic energy in each simulation. Here, we define $\mathcal{E}_{B}$ to be the initial energy in the total magnetic field, which includes both the reversing field and the guide field, and $\Delta \mathcal{E}_{B}$ is the change of the total magnetic energy from the beginning to the end of the simulation. The energy in the reversing field only is a factor of $\approx\left(1+\kappa^{2}\right)^{-1}$ smaller than $\mathcal{E}_{B}$ if we ignore the reduction of the reversing field in the current sheets. Independent of this correction, $\left|\Delta \mathcal{E}_{B}\right| / \mathcal{E}_{B}$ exhibits a strong decreasing trend with increasing guide field strength $\kappa$. It also exhibits a weaker increasing trend with $\sigma$; note that the very high $\left|\Delta \mathcal{E}_{B}\right| / \mathcal{E}_{B}$ in simulation $\mathrm{S} 1 \mathrm{KO}$ is the result of destructive current sheet interaction. In the simulations with $\kappa \geqslant 0.5$, and also in simulation S1K025 with $\kappa=0.25$ and $\sigma=1$, the conversion of magnetic to kinetic energy is substantially weaker than in the other simulations. We also note that the rate of development and evolution of the flux rope network exhibits similar behavior in the sense that flux ropes of a given size assemble later $\left(\sim 80 \tau_{c 0}\right.$ versus $\left.\sim 40 \tau_{c 0}\right)$ in the $\kappa \geqslant 0.5$ and $\mathrm{S} 1 \mathrm{~K} 025$ simulations.

The variation of $\left|\Delta \mathcal{E}_{B}\right| / \mathcal{E}_{B}$ and of the overall evolution rate with $\kappa$ is likely a consequence of the decrease of plasma compressibility with increasing guide field strength (e.g., Zenitani \& Hesse 2008 and references therein). Since most of the magnetic to particle kinetic energy conversion takes place during the relaxation of reconnection field lines to their final equilibrium in flux ropes, the less compressible case of a strong guide field leads to larger flux ropes and smaller energy conversion.

\subsubsection{Energization Efficiencies}

Figure 7, top panel, shows the energy spectrum at a reference time $t=150 \tau_{c 0}$ in six of the smaller size simulations. Each of the spectra exhibits a peak in agreement with the initial thermal distribution as well as a new tail resulting from particle energization extending to maximum Lorentz factors in the range $\gamma_{\max } \sim 30-50$ (for the precise values at the end of the simulations, see Table 1). The simulations with the strongest particle energization are those with higher magnetization $\sigma=2$ and zero or moderate guide field $\kappa \leqslant 0.25$. Rather weak energization is seen in all simulations with strong guide field $\kappa=1$ and in the simulation S1K025 with weak magnetization and moderate guide field. We observe an intermediate level of energization in simulation S1K0 with weak magnetization and no guide field; this simulation is unique in that at the reference time, the two current sheets have already interacted with each other. It is important to note that all of these spectra are calculated at the same reference time; therefore, these differences in energization efficiency might result from the differing energy conversion rates discussed in the previous section. However, we will find below that the spectrum exhibits the strongest evolution during the flux rope merging phase; because the merging phase is complete by the reference time in all the simulations, it seems that the differences in the level of energization between the runs cannot be attributed solely to the differences in the rate of reconnection.

In order to quantify the degree of particle energization in the simulations, following Zenitani \& Hoshino (2007),

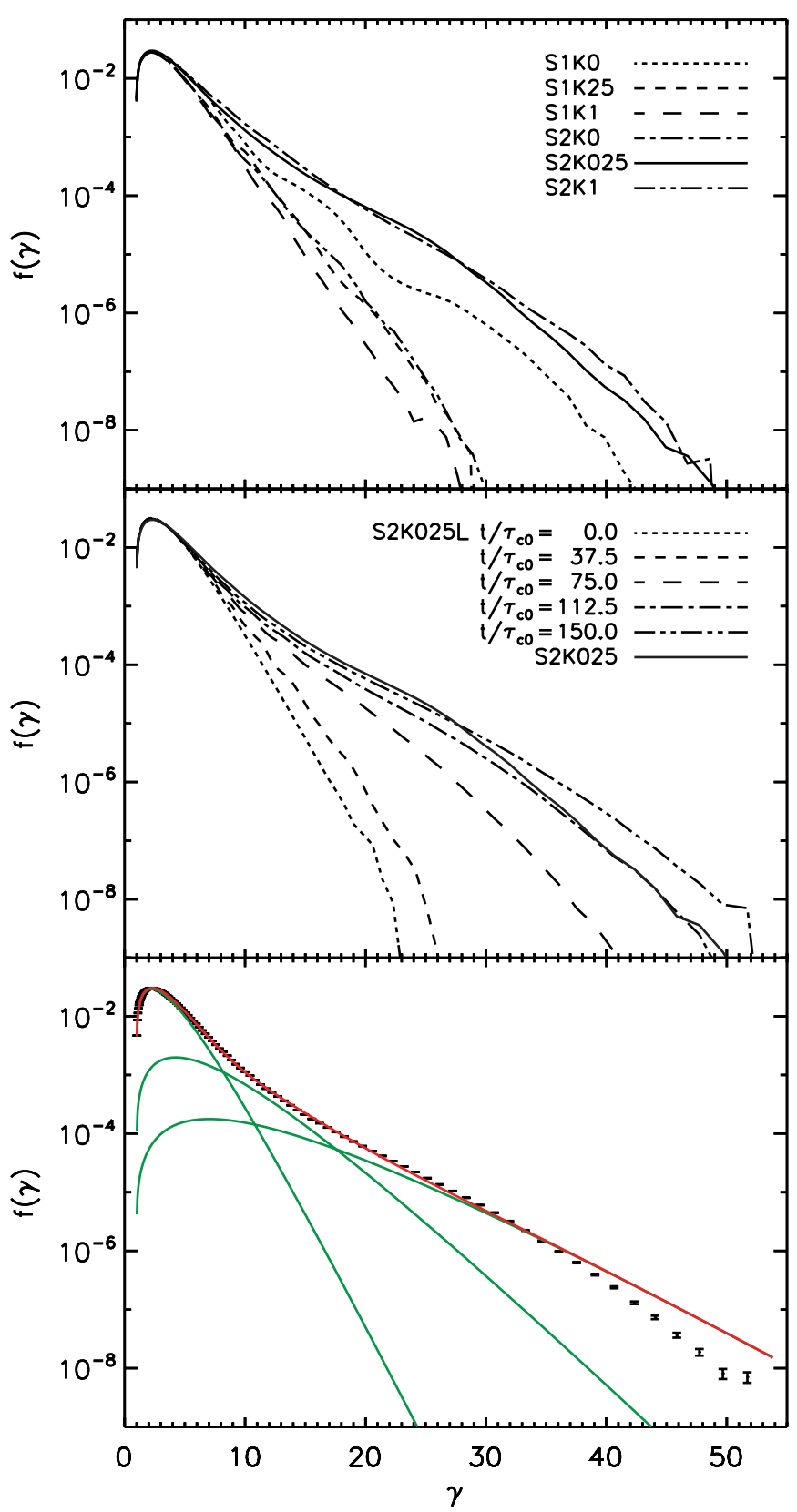

Figure 7. Particle energy spectra in the six smaller size simulations at time $t=150 \tau_{c 0}$ (top panel; see legend) and in the large size simulation S2K025L at five different times (middle panel; see legend) where for reference we include the $t=150 \tau_{c 0}$ spectrum from the corresponding smaller size simulation S2K025 (solid line). The bottom panel compares the final spectrum to a model containing three thermal populations at different temperatures (red line; see text), including the spectra of the three individual thermal components (green lines). The spectra and uncertainties are calculated from a random sample containing $5 \%$ of the particles in the simulation.

(A color version of this figure is available in the online journal.)

we compute the particle kinetic energy $K_{\text {ener }}$ contained in the difference between the measured spectrum and a thermal spectrum containing the same number of particles and the same energy at particle energies at which the measured spectrum is in excess of the thermal spectrum. We then calculate the ratio of $K_{\text {ener }}$ to the total particle kinetic energy $K$, both computed at the end of the simulation. It is worth remarking that we do not go as far as Zenitani \& Hoshino $(2007,2008)$ to identify the particles contributing to $K_{\text {ener }}$ as a nonthermal population; in the following section, we show this tail may be better described 
with a combination of several thermal populations. Consistent with the trend observed in the shape of the spectrum, we find that $K_{\text {ener }} / K$, which is shown in Table 1 , is the largest in the simulations with the higher magnetization $\sigma=2$ and at most moderate guide field $\kappa \leqslant 0.25$ and is the smallest in simulation S1K1 with lower magnetization $\sigma=1$ and strong guide field $\kappa=1$. Thus, in contrast with Zenitani \& Hoshino (2008), we find that $K_{\text {ener }} / K$ decreases with increasing guide field. The reason for this dissimilarity seems to be that the kink instability does not compromise the development of reconnection in any of our simulations other than S1K0; even in that run, a minor flux rope merger occurs before the current sheet is disrupted. Without the kink instability, more particle energization can take place.

Another possibly more interesting measure of particle energization is the ratio of the kinetic energy in energized particles to the magnetic energy converted to kinetic energy. Combining Equations (1) and (6) with the definition of $\Gamma$, and taking into account the conservation of energy $K(t)=K(0)+\left|\Delta \mathcal{E}_{B}\right|$, this ratio can be written as

$$
\frac{K_{\text {ener }}}{\left|\Delta \mathcal{E}_{B}\right|}=\left(\xi\left|\frac{\Delta \mathcal{E}_{B}}{\mathcal{E}_{B}}\right|^{-1}+1\right) \frac{K_{\text {ener }}}{K} .
$$

Here, the dimensionless coefficient $\xi$ is the ratio of the initial particle kinetic energy density to the magnetic energy density including the guide field, given by

$$
\xi \approx \frac{1}{\sigma\left(1+\kappa^{2}\right)}\left(\frac{1}{\Gamma-1}-\frac{1}{\theta_{0}}\right),
$$

where $\theta_{0} \equiv T_{0} /\left(m_{e} c^{2}\right)$ and all the quantities entering the definition of $\xi$ are evaluated at the beginning of the simulation. The ratio, which ranges between $11 \%$ and $38 \%$, is shown in Table 1. The trend seen at low magnetization $\sigma=1$ is an increase of $K_{\text {ener }} /\left|\Delta \mathcal{E}_{B}\right|$ with guide field strength, approximately the opposite of that seen in $K_{\text {ener }} / K$. The trend can be understood by noting that, as we will find in Section 3.7.3, most of the particle energization contributing to $K_{\text {ener }}$ occurs in X-line regions. Meanwhile, $\Delta \mathcal{E}_{B}$ measures the change in magnetic energy both during reconnection in $\mathrm{X}$-line regions and, more importantly, during the subsequent contraction of reconnected magnetic field lines into flux ropes. Therefore, the ratio $K_{\text {ener }} /\left|\Delta \mathcal{E}_{B}\right|$ should indeed increase with stronger guide field because the guide field reduces plasma compressibility and moderates field line contraction.

\subsubsection{Energy Spectrum Evolution and Structure}

Figure 7, middle panel, shows the evolution of the particle energy spectrum in the large simulation S2K025L. The spectrum evolves little during the linear tearing phase and the first round of flux rope merging at $t<37.5 \tau_{c 0}$, but then it quickly hardens by $t=75 \tau_{c 0}$ as the flux rope merging concludes and the reconnection layer transitions to a disordered state. Further hardening takes place until the end of the simulation at $t=150 \tau_{c 0}$. For comparison, we also show the particle energy spectrum at this time from the smaller, equivalent simulation S2K025. The two spectra are consistent at Lorentz factors $\gamma \lesssim 28$, indicating convergence with increasing box size at relatively low energies. However, the larger simulation has progressively more particles at still higher energies, with Lorentz factors reaching $\gamma \approx 50$.

We proceed to model the entire spectrum in simulation S2K025L as is, while keeping in mind that in a still larger simulation, further evolution of the spectrum at the highest energies is likely to be expected. We experimented with composite populations containing thermal as well as power law components, and found that at Lorentz factors $\gamma \lesssim 35$, a model containing three thermal populations, each described by a relativistic Maxwellian, seems to work best. The model and the three thermal components are shown in the bottom panel of Figure 7. We fix the temperature of the first population to be equal to the temperature of the initial plasma, $T_{1}=m_{e} c^{2}$. A least-squares fit yielded temperatures $T_{2}=2.1 m_{e} c^{2}$ and $T_{3}=3.5 m_{e} c^{2}$ for the second and third population, respectively. The first component contains $85 \%$ of the particles and $70 \%$ of particle kinetic energy, the second component contains $13 \%$ of particles and $24 \%$ of particle kinetic energy, and the third component contains only $2 \%$ of the particles and $6 \%$ of particle kinetic energy. We show in Section 3.7.3 that the energized populations, and especially the particles with $\gamma>30$, are located close to the primary $\mathrm{X}$-lines (within about one Larmor radius) and in flux ropes.

\subsubsection{Energization Sites and Mechanism}

To gain insight into the nature of the particle energization process, in simulation $\mathrm{S} 2 \mathrm{~K} 025$ we select a number, $N_{\text {trace }}=$ 1841 , of particles reaching the highest energies, corresponding to Lorentz factors $\gamma \geqslant 32$, at the end of the flux rope merging phase at $t=75 \tau_{c}$. We trace the orbits of these particles throughout the merging phase over the time interval $47 \tau_{c} \leqslant t \leqslant 75 \tau_{c}$. This is the period during which the particles experience coherent energization. The particles generally begin in the current sheet and have momenta that, on average, are aligned with the direction of the electric force $q_{j} \mathbf{E}$ in the current sheet, with a median inclination of $\approx 30^{\circ}$ from the direction of the force.

During flux rope merging, the traced particle energies increase approximately linearly. However, at the instance of merging for the two largest flux ropes, which occurs at $t \approx 61 \tau_{c}$, most of the particles incur an energy increment accounting for $\approx 15 \%-20 \%$ of the total energy gain. Following the flux rope merging phase, some traced particles gain additional energy, but others lose energy, and both the gain and the loss could be considered manifestations of a thermalization process. Indeed, the energization $K_{\text {ener }} / K$ does not further increase after the flux rope merging is complete.

To pin down the geometric location of particle energization, for the traced particles, we compute the total energy gain per particle per unit volume averaged over $y$ via

$$
\begin{aligned}
\Upsilon(x, z)= & \frac{1}{N_{\text {trace }}} \sum_{j=1}^{N_{\text {trace }}} \frac{q_{j}}{m_{e} c^{2}} \int \mathbf{v}_{j}(t) \cdot \mathbf{E}\left[\mathbf{x}_{j}(t)\right] \\
& \times \delta\left[\mathbf{x}-\mathbf{x}_{j}(t)\right] d t \frac{d y}{L_{y}}
\end{aligned}
$$

where $\mathbf{x}_{i}(t)$ and $\mathbf{v}_{i}(t)$ are the position and velocity of traced particle $j$ at time $t, \delta$ is the Dirac delta, and the time integral covers the flux rope merging phase. In Figure 8, we compare the energy gain $\Upsilon$ to the corresponding absolute value of the $y$ - and $t$-averaged current density $J_{y}$. The highest energy gain is clearly associated with the largest, best-defined X-line on the lower left. Substantial energization is also detected in the outflow regions flanking this X-line. The total energy gain in the flanking regions, seen vertically above and below the $\mathrm{X}$-line in the figure, is larger than that in the narrow vicinity of the $\mathrm{X}$-line. 


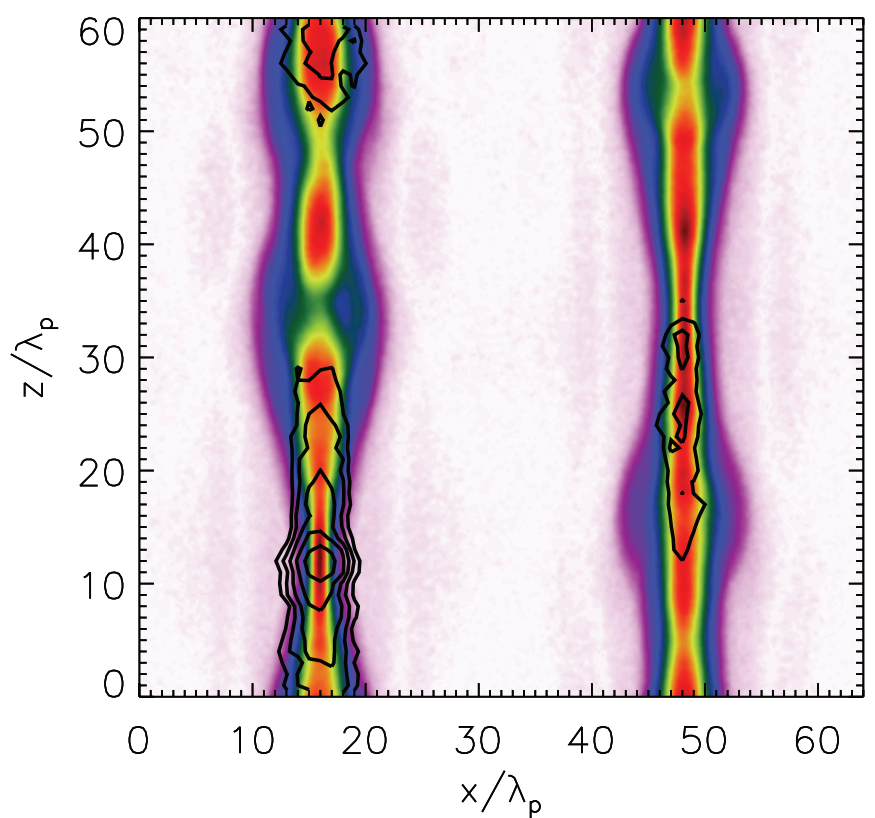

Figure 8. The locus of particle acceleration in simulation $\mathrm{S} 2 \mathrm{~K} 025$ in the interval $47 \tau_{c} \leqslant t \leqslant 70 \tau_{c}$ corresponding to the primary particle energization phase. The contours show the logarithm of the $y$-averaged energy gain per particle per unit volume $\Upsilon(x, z)$ for the $\approx 2000$ highest-energy particles in the simulation in this interval (see text); the lowest contour corresponds to 0.04 times the peak value of $\Upsilon$. The underlying color image shows the projection in the $x z$ plane of the current density $J_{y}$ averaged over the interval; the current density is scaled linearly from light purple to dark red.

(A color version of this figure is available in the online journal.)

It is puzzling that the overall energization efficiency is weaker in the second current sheet shown on the right in the figure. In both sheets, the energization seems to be associated with some current density maxima but not with others. Energization seems to prefer long, continuous X-line regions with thin current sheets, perhaps because these are the sites of plasma inflow.

In Figure 9, we show the orbits of six representative traced particles belonging to the current sheet shown on the left of Figure 8 . They all start in the current sheet within $\sim 8 \lambda_{\mathrm{p}}$ from the $\mathrm{X}$-line and have initial Lorentz factors in the range $\gamma \sim 16-23$. While in the $\mathrm{X}$-line region, the particles oscillate across the current sheet on Speiser-like orbits. The particles reach Lorentz factors of $\gamma \sim 32$ (this is how they were selected; see Figure 9, right panel) after drifting from the $\mathrm{X}$-line region into the neighboring islands. At the final Lorentz factor, the particles have Larmor radii evaluated using the reversing magnetic field $r_{\mathrm{L}}=\gamma m_{e} c^{2} /\left(e B_{0}\right) \sim 17 \lambda_{\mathrm{p}}$, larger than the width of the flux ropes in the simulation. Because the magnetic field in flux ropes is only somewhat enhanced compared to the reversing field, the accelerated particles are not easily trapped within the flux ropes.

The electric field is nearly uniform across the reconnection region, hence the electric force acting on a particle trapped in a Speiser orbit is approximately constant in time provided that the inclination of the particle's velocity relative to the direction of the electric force is relatively small. Most of the traced particles happen to fulfill this condition. Then, the work done by the electric force on the particle is independent of the initial particle energy. We have also checked that, we expect for the Speiser orbit acceleration, it is the $y$-component of the electric field that contributes the most to the energy gain. The average total energy gain per particle obtained by integrating $\Upsilon$ over the volume of the simulation is $\Delta \gamma \approx 12$.

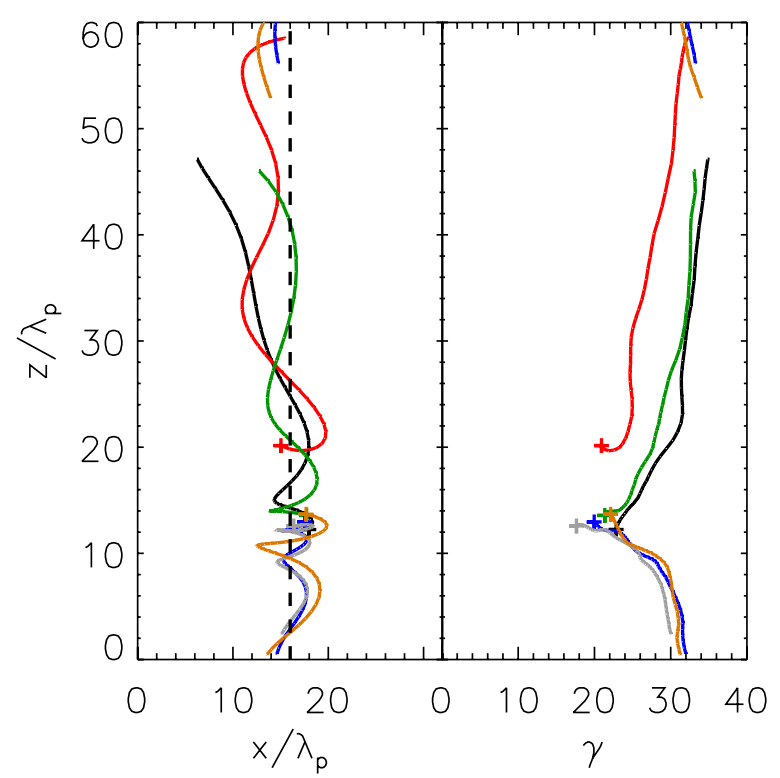

Figure 9. The trajectories of six representative particles that attain Lorentz factors $\gamma>30$ in the simulation S2K025 (left panel), and the evolution of the Lorentz factor as a function of coordinate $z$ (right panel). The dashed line indicates the center of the current sheet. Note that many of the particles start at $z \approx 12.5 \lambda_{\mathrm{p}}$ and close to the center of the current sheet; this is the location of the large X-line on the lower left in Figure 8. The crosses indicate the particle position at the beginning of the acceleration phase.

(A color version of this figure is available in the online journal.)

Because the rate of particle acceleration in the $y$-direction is constant in time, the total energy gain is limited by the time the particle spends in the X-line region (see, e.g., Cerutti et al. 2012a). Particles displaced from the very center of the X-line experience a Lorentz force due to the reconnected magnetic field $B_{x}$ that deflects them away from the X-line and toward the flux rope. Once a particle has left the $\mathrm{X}$-line region and entered a flux rope, it no longer experiences coherent acceleration.

While Drake et al. (2006) found that acceleration internal to flux ropes occurred when the guide field was present, this mechanism has not typically been important in other works for the guide field case (Fu et al. 2006; Huang et al. 2010). We note that unlike the particles traced in Drake et al. (2006), our energized particles already have very large Larmor radii by the time they transition from the $\mathrm{X}$-line region into a flux rope and the magnetic geometry and dynamics also seem markedly different.

\subsubsection{Angular Distribution of the Highest-Energy Particles}

If the motion of high energy particles accelerated in a reconnection region is anisotropic, then the synchrotron radiation emitted by these particles carries angular dependence. Radiation emitted by beams of accelerated particles can come in and out of view of an observer; at any given time, the observer detects only the radiation emitted by particles with momenta making angles $\lesssim \gamma^{-1}$ from the line of sight. Such beaming could explain the temporal variability often seen in astrophysical sources, such as gamma-ray bursts (e.g., Zhang \& Yan 2011; McKinney \& Uzdensky 2012) and blazars (e.g., Nalewajko et al. 2011), that may be powered by reconnection. It could also influence the characteristics of the gamma-ray emission from other candidate reconnection-powered astrophysical sources, such as magnetars (Thompson \& Duncan 1995; Lyutikov 2003; Parfrey et al. 2012) and pulsar wind nebulae (e.g., Lyubarsky \& Kirk 2001; Sironi \& 

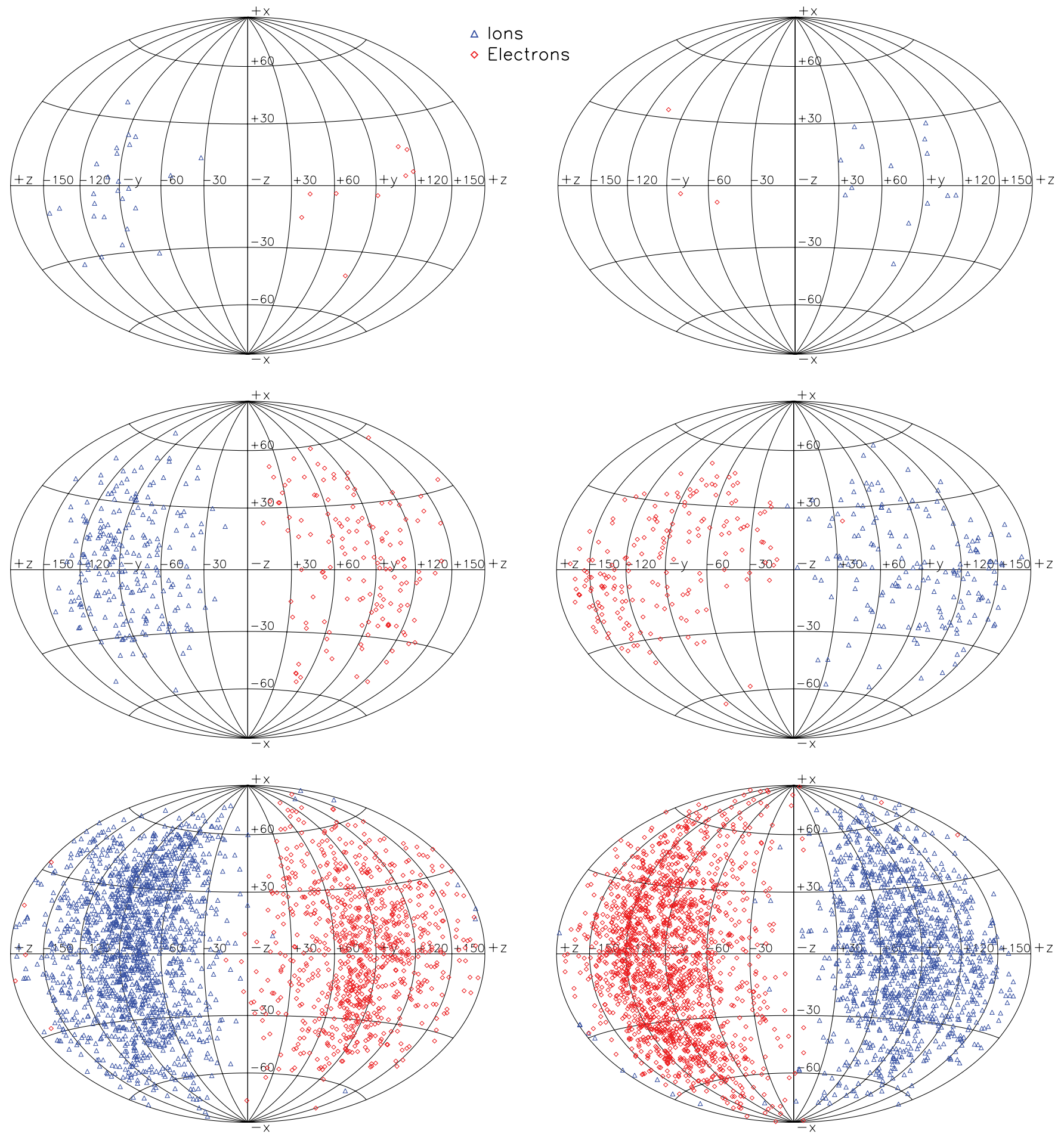

Figure 10. Angular distribution of the momenta of particles with Lorentz factors $\gamma \geqslant 30$ at times $t=56 \tau_{c 0}$ (top panels), $t=66 \tau_{c 0}$ (middle panels), and $t=84 \tau_{c 0}$ (bottom panels) in simulation S2K025L shown in the Aitoff projection. The panels of the left and right show the particles near the current sheets at $x=L_{x} / 4$ and $x=3 L_{x} / 4$, respectively. The plotted particles were selected from a random sample containing $5 \%$ of the particles in the simulation.

(A color version of this figure is available in the online journal.)

Spitkovsky 2011; Cerutti et al. 2012a). We investigate the angular distribution of the momenta of the highest-energy particles in our simulation, and compare with the results of Cerutti et al. (2012b), who recently reported a high degree of beaming in a two-dimensional PIC simulation of pair plasma reconnection initialized with $\sigma \sim 40$.

Figure 10 shows the orientations of the momenta of all of the electrons and ions with $\gamma \geqslant 30$ in simulation S2K025L.
We select the larger simulation for this analysis to include a larger range of three-dimensional effects to which the particle anisotropy may be sensitive. The median inclination of electrons (ions) to the $y$-axis (negative $y$-axis) is $\sim 30^{\circ}-40^{\circ}$, and hence half of the particles in each charge species occupy a fraction $\Delta \Omega /(4 \pi) \sim 0.06-0.12$ of the full solid angle. This moderate degree of beaming is a natural consequence of X-line acceleration by the electric field in the reconnection region, since the 
electric field is uniform and accelerates the particles preferentially along the $y$-axis.

The degree of beaming, however, is much smaller than in Cerutti et al. (2012b), where the highest-energy particles, with Lorentz factors $\gamma>40$, were strongly beamed, occupying a solid angle fraction as small as $\Delta \Omega /(4 \pi) \sim 0.01$. The dependence of the beaming on the parameters of the reconnection layer can crudely be understood as follows. Assuming for simplicity that the plasma is ultrarelativistic and highly magnetized, and that the energy of the accelerated particle increases by a large factor, the components of its momentum following acceleration are approximately $p_{y} \sim \Delta \gamma m_{e} c$ and $p_{x} \sim p_{z} \sim \gamma_{\text {init }} m_{e} c$, where $\gamma_{\text {init }}$ is the initial Lorentz factor of an accelerated particle, and $\Delta \gamma$ is the Lorentz factor gain during acceleration, which increases the particle momentum in the $y$ direction. Therefore, the particle's degree of beaming is proportional to $\Omega /(4 \pi) \propto\left(p_{x}^{2}+p_{z}^{2}\right) / p_{y}^{2} \propto\left(\Delta \gamma / \gamma_{\text {init }}\right)^{-2}$. Using $\Delta \gamma \propto e E \Delta t /\left(m_{e} c\right)$, where $E \sim r_{\text {rec }} v_{\mathrm{A}} B_{0} / c$ is the accelerating electric field (Equation 25) and $\Delta t \sim \tau_{\text {merge }} \sim \Delta z / v_{\mathrm{A}}$ is the duration of acceleration (Equation 19), and expressing the magnetic field in terms of the magnetization parameter $B_{0}^{2} \propto \sigma n_{0} T_{0} \propto \sigma\langle\gamma\rangle \theta_{0}\left(m_{e} c^{2}\right)^{2} /\left(e \lambda_{\mathrm{p}}\right)^{2}$, where $\theta_{0} \equiv T_{0} /\left(m_{e} c^{2}\right)$ (Equations 6 and 7), we obtain

$$
\frac{\Omega}{4 \pi} \propto \frac{1}{\sigma}\left(\frac{\gamma_{\text {init }}^{2}}{\theta_{0}\langle\gamma\rangle}\right)\left(r_{\mathrm{rec}} \frac{\Delta z}{\lambda_{\mathrm{p}}}\right)^{-2} .
$$

This result is accurate in the regime $\theta_{0} \gtrsim 1$, whereas Cerutti et al. set up their simulation with $\theta_{0}=0.15$. Ignoring this concern, we estimate that the factors in parentheses on the righthand side of Equation (30) are similar in our simulation and in Cerutti et al. We therefore expect that the beaming solid angle $\Omega /(4 \pi)$ in their simulation should be smaller by the inverse ratio of the magnetizations in the two simulations, which is $\left(\sigma_{\text {Cerutti }} / \sigma_{\mathrm{S} 2 \mathrm{~K} 025 \mathrm{~L}}\right)^{-1}=0.05$. Thus, the crude expectation is that the particles accelerated in Cerutti et al. should be more beamed within a solid angle over an order of magnitude smaller than in our simulation, consistent with the observed beaming ratio between the two simulations. In conclusion, this analysis suggests that the high magnetization and large physical size of the $\mathrm{X}$-line region can both give rise to a high degree of beaming.

\section{DISCUSSION}

\subsection{Tearing, Kink, and Oblique Modes}

A number of studies have examined the three-dimensional evolution of a current sheet without a guide field; here, we focus on results relevant to pair plasmas. Zenitani \& Hoshino (2008) found that the kink mode dominated the tearing mode in antiparallel reconnection. In contrast, Liu et al. (2011) found that the initial evolution was dominated by the tearing mode, leading to an initial merging phase similar to that in two dimensions. After the completion of the merging phase, however, they found that a secondary kink instability set in and rendered the reconnection region turbulent and filled with plasmoids covering a range of dimensions. The reduced influence of the kink mode in the simulations of Liu et al. (2011) may be explained by initially relativistic drift velocities of $0.82 c$. Analytical calculations suggest that while the kink mode dominates at low drift velocities, the tearing mode becomes dominant for drift velocities exceeding $0.6 c$ (Zenitani \& Hoshino 2007). However, the growth rate of the kink mode, unlike the tearing mode, depends strongly on the initial structure of the current sheet (Daughton 1999).

In guide field reconnection, previous three-dimensional studies have found that the kink mode is stabilized, but, under certain conditions, oblique modes dominate the evolution. Zenitani \& Hoshino (2008) found that with a guide field present, the tearing mode and a sausage-like mode are combined into an oblique "relativistic-drift-sausage-tearing" mode. Daughton et al. (2011) found that in electron-ion plasmas, oblique modes give rise to a network of interconnected flux ropes. In both cases, the nonlinear development of the oblique modes led to turbulence in the current sheet. In nonrelativistic electron-ion simulations with a strong guide field, Che et al. (2011) found that at low temperatures, the current sheet filamented and that the turbulent transport played a key role in the diffusion of the field. At higher, albeit still nonrelativistic temperatures, their filamentation instability became weaker and the current sheet did not become turbulent.

One must be aware of the possibility that unlike the tearing mode, the other instabilities identified in simulations starting with Harris sheet equilibria are idiosyncrasies of the initial configuration and have little to do with astrophysical magnetic reconnection. Our simulation, starting from a non-Harris like configuration lacking a density enhancement in the initial current sheet, should help discern artifacts of the initial configurations from genuine properties of reconnection dynamics.

We have found that in our simulations, in which we considered guide fields at most equal to the reversing field, the tearing mode dominates the evolution at all guide field strengths except in Run S1K0. To understand the difference between our simulations without guide field and the studies that detected a dominant kink mode, we have carried out two-dimensional simulations with $\sigma=2$ and $\kappa=0$ in both the $x y$ and $x z$ planes. We find that the kink mode grows faster in a Harris current sheet, while the tearing mode grows faster starting with our initial conditions. Our initial configuration seems to enhance the growth rate of the tearing mode and inhibit the growth of the kink mode. The plasma inflow occurring during readjustment to equilibrium may enhance growth of the tearing mode above the value expected in pressure equilibrium. Furthermore, our initial configuration may be endowed with a somewhat stronger drift velocity shear than the Harris sheet and this could stabilize the kink mode (Volponi et al. 2000) while having little effect on the tearing mode (Roytershteyn \& Daughton 2008).

In our simulations with guide field, we do not find any oblique modes similar to those observed by Zenitani \& Hoshino (2008) or Daughton et al. (2011). Therefore, the only linear mode present is the tearing mode; the lack of phase coherence is the mechanism responsible for breaking translational symmetry. Because this mechanism is a general result of causal constraints, it is likely that this mechanism is present in Harris current sheets as well as in our simulations, but is masked by the more rapid effects of kink and oblique modes.

We proceed to calculate the theoretical and actual tearing mode growth rates in each simulation. In Table 2, we show the two rates which we label $\omega_{\mathrm{KTI}, \mathrm{t}}$ and $\omega_{\mathrm{KTI}, \mathrm{s}}$, along with the initial particle drift velocity $\beta_{0}$, the initial current sheet compression factor $f_{\mathrm{c}}$, and the number of flux ropes per current sheet determined by visual inspection $N_{\mathrm{FR}}$. Because the fastestgrowing tearing wavelength computing from the theoretical result in Equation (12) is $\sim 10.8 \lambda$ and the box size in all but simulation S2K025L is $20 \lambda_{0}$, the number of flux ropes per current sheet produced by the tearing mode should be 
Table 2

Tearing Instability Parameters

\begin{tabular}{lccccc}
\hline \hline Run & $\beta_{0}$ & $f_{\mathrm{c}}^{\mathrm{a}}$ & $N_{\mathrm{FR}}{ }^{\mathrm{b}}$ & $\tau_{c 0} \omega_{\mathrm{KTI}, \mathrm{t}}{ }^{\mathrm{c}}$ & $\tau_{c 0} \omega_{\mathrm{KTI}, \mathrm{s}}{ }^{\mathrm{c}}$ \\
\hline S1K0 & 0.365 & 1.5 & 3 & 0.12 & 0.06 \\
S1K025 & 0.365 & 1.0 & 2 & 0.08 & 0.04 \\
S1K1 & 0.365 & 1.0 & 2 & 0.08 & 0.03 \\
S2K0 & 0.344 & 2.3 & 4 & 0.16 & 0.14 \\
S2K025 & 0.344 & 2.3 & 4 & 0.16 & 0.13 \\
S2K025L & 0.344 & 2.3 & 9 & 0.16 & 0.11 \\
S2K1 & 0.344 & 2.3 & 2 & $0.09^{\mathrm{d}}$ & 0.07 \\
\hline
\end{tabular}

\section{Notes.}

${ }^{\mathrm{a}} f_{\mathrm{c}}$ is the ratio of the original current sheet width to the current sheet width after the readjustment phase.

b $N_{\mathrm{FR}}$ is the number of flux ropes per current sheet initially formed in the simulation.

c The two values of $\tau_{c 0} \omega_{\mathrm{KTI}}$ are the normalized theoretical (subscript "t") and simulated (subscript "s") growth rates of the tearing mode.

$\mathrm{d}$ This growth rate is based on the observed mode wavelength, which is significantly longer than the fastest-growing wavelength.

$N_{\mathrm{FR}}=1.9 f_{\mathrm{c}}$; the number should be twice as large in S2K025L. The actual number of flux ropes formed in the simulation is in agreement with this prediction, with the exception of the simulation S2K1 with the strongest guide field. This run produced only two flux ropes per current sheet even though one expects four. As discussed in Daughton et al. (2011), the strong guide field may prefer oblique rather than pure tearing modes, but the former grow the fastest at long wavelengths $\sim 33 \lambda_{\mathrm{p}}$, about half the box size of the simulation, and the finite box size interferes with ability of arbitrary oblique modes to grow.

The values of $\omega_{\text {KTI,t }}$ for the various runs are shown in Table 2; it should be noted that these are upper limits to the possible tearing mode growth rates, because any measured growth rates are found from estimates in the nonlinear regime. We also compute the simulated growth rates $\omega_{\mathrm{KTI}, \mathrm{s}}$ of the tearing mode in our simulations by calculating the growth rate of the average reconnected magnetic field perturbation $B_{x}$ in the current sheet. Growth rates of the KTI calculated with the latter method should be higher than those obtained by examining a single Fourier mode, as we did in Section 3.3, because multiple Fourier modes contribute to the amplitude of the perturbation. We find that the theoretical and simulated growth rates were relatively similar in all simulations, with theoretical rates being at most double the simulated rates. Zenitani \& Hoshino (2007) identified a similar discrepancy between the theoretical and simulated growth rates. The growth rates also show that decreased guide field $\kappa$, and especially higher magnetization $\sigma$, lead to higher tearing mode growth rates both in theoretical calculations and in the simulations. This reinforces the effects of the variation in $N_{\mathrm{FR}}$, which has a similar dependence on $\sigma$ and $\kappa$.

We have shown that the tearing mode is responsible for producing magnetic flux ropes in most of our simulations. The tearing mode can also explain many of the differences between the simulations. The runs with higher $\sigma$ and smaller $\kappa$ have larger values of $f_{c}$, which leads to higher tearing mode growth rates and the formation of a larger number of flux ropes $N_{\mathrm{FR}}$. The faster evolution of runs with higher $\sigma$ and smaller $\kappa$ can be explained by the faster growth of the tearing mode in such runs. Because runs with higher $\sigma$ and smaller $\kappa$ are also associated with a larger $N_{\mathrm{FR}}$, more flux rope merging can take place in such runs. Flux rope merging is strongly associated with energy transfer and particle acceleration in our simulations, so the larger energy transfer in runs with higher $\sigma$ and smaller $\kappa$ can be explained by the larger $N_{\mathrm{FR}}$ in those runs. This suggests that a large portion of the variation between runs is a result of the effect of $\sigma$ and $\kappa$ on the tearing mode.

\subsection{Particle Energization}

Various particle energization channels have been identified in PIC simulations of plasma reconnection (see, e.g., Oka et al. $2010 \mathrm{~b}$ and references therein). Three specific loci where particle energization was detected include: inside or near the diffusion region containing reconnection X-lines; in the magnetic islands (or plasmoids, flux ropes) where the reconnected magnetic flux accumulates; and between an X-line and the edge of a flanking island, where the plasma flowing out of the X-line first encounters a strong magnetic field gradient.

A number of studies point to the conclusion that significant energization occurs near the primary (i.e., largest, or highest rank) X-lines. There, the electric field, which is perpendicular to the reversing field and is aligned with the current density vector in the middle of the current sheet, can accelerate particles oscillating within or across the current sheet (e.g., Zenitani \& Hoshino 2001, 2007, 2008; Lyubarsky \& Liverts 2008; Uzdensky et al. 2011; Bessho \& Bhattacharjee 2012; Cerutti et al. 2012a, 2012b). A variant of this mechanism involves the trapping of particles in secondary magnetic islands appearing within the diffusion region of an X-line (Oka et al. 2010a); we will not discuss this as we do not observe secondary flux rope formation in our simulations.

Particles can also be accelerated outside of the X-lines, such as in primary magnetic islands. If an island contracts in the course of its relaxation to MHD equilibrium, then the particles trapped inside it can be accelerated by a Fermi-type process (e.g., Drake et al. 2006, 2010; Kowal et al. 2011). Yet another location for particle acceleration is in the pileup region between the X-line and a flanking island, which is where the reconnected magnetic flux accumulates. There, relativistic Speiser motion can combine with curvature drift along the magnetic field gradient to create significant acceleration (e.g., Hoshino et al. 2001; Jaroschek et al. 2004; Zenitani \& Hoshino 2007; Pritchett 2008; Huang et al. 2010; Liu et al. 2011).

These mechanisms can operate in reconnection sites where the plasma configuration, involving inflow into the diffusion region and outflow toward the flanking islands, is relatively stationary, or inside a single, autonomously evolving magnetic island. There may be other mechanisms that operate in the course of spatial rearrangement and the merging of magnetic islands. Converging islands can give rise to Fermi-type acceleration as particles bounce between them (e.g., Oka et al. 2010b; Tanaka et al. 2011). A dynamically active reconnection region containing many interacting islands can also allow Fermi-type stochastic particle acceleration (Drake et al. 2010; Hoshino 2012). It is worth noting that even if particle acceleration is not directly driven by the merging, it is normally most active during island merging episodes in the course of a reconnection event (Jaroschek et al. 2004; Pritchett 2008).

The presence of a guide field has a significant effect on particle acceleration in reconnection (Zenitani \& Hoshino 2008). Without a guide field, the direct X-line acceleration was typically found to be less efficient than the other mechanisms discussed. However, with a guide field, the effectiveness of acceleration in the pileup region (Huang et al. 2010) and inside magnetic islands (Fu et al. 2006) was diminished, thus leaving X-line 
acceleration of particles on Speiser orbits as the dominant mechanism. Our results are consistent with this conclusion.

To permit an interpretation of the nonthermal radiation spectra in observed high-energy astrophysical sources in terms of synchrotron and inverse-Compton radiation, a relatively hard power law particle energy spectrum, which places a significant portion of the total energy in high-energy particles, is required. In most PIC simulations, including the present work, reconnection produced energized particle populations, but whether or not the populations represented genuine power law tails has remained a matter of interpretation. Alternatively, an energized population can be interpreted as a composite of one or more thermal sub-populations, each at a different temperature, for example. Two-dimensional simulations typically provide the dynamic range to make a tentative distinction between a thermal or a power law spectrum in an energized population. In most three-dimensional simulations, however, such a determination is dubious. In what follows, we discuss the characteristics of the particle energy spectra in the literature and provide a comparison with our results. Then, we briefly reflect on the expected nature of particle energy spectra produced by systems experiencing magnetic reconnection.

Most investigations involving PIC simulations of plasma reconnection have interpreted a section of the high energy tail of the particle energy spectrum as a power law $d N / d \ln \gamma \propto \gamma^{\alpha}$. In the X-line region of two-dimensional simulations, spectra with power law indices as hard as $\alpha \approx-1$ have been reported (Zenitani \& Hoshino 2001, 2007; Jaroschek et al. 2004; Bessho \& Bhattacharjee 2007; Lyubarsky \& Liverts 2008). The spectrum in the whole simulation box also contains a power law component, but with a softer index of $\alpha \sim-2.5$. In their two- and three-dimensional simulations of shock-induced reconnection, Sironi \& Spitkovsky (2011) find that as long as the region containing a reversing magnetic field that can undergo reconnection is reasonably large compared to the thickness of the reconnection layer, the particle energy spectrum at high energies is a power law with an index $\alpha=-1.5$ over a decade in energy. Other three-dimensional investigations have identified relatively soft power law spectra with indices $\alpha \sim-3$ both in the $\mathrm{X}$-line region and in the entire simulation box (Jaroschek et al. 2004; Zenitani \& Hoshino 2008).

Other investigations have interpreted particle energy spectra in terms of multiple thermal and other exponentially truncated populations (e.g., Oka et al. 2010b). In their two-dimensional simulation of reconnection in an initially nonrelativistic plasma at temperature $T=0.15 m_{e} c^{2}$, Cerutti et al. (2012b) find a new thermal population at temperature $T \sim 4 m_{e} c^{2}$. In a threedimensional simulation beginning with a relativistic plasma at temperature $T=m_{e} c^{2}$, Liu et al. (2011) find that a thermal population with $T \approx 2.3 m_{e} c^{2}$ is produced. In a recent work presenting two-dimensional simulations without a guide field, Bessho \& Bhattacharjee (2012) detect a spectrum of the form $d N / d \gamma \propto \gamma^{-1 / 4} \exp \left(-a \gamma^{1 / 2}\right)$, where $a$ is a constant of the order of unity.

Among the cited descriptions of particle populations energized by reconnection, the spectra observed in our simulations bear resemblance with those involving multiple thermal subpopulations. For example, the spectrum at the end of simulation S2K025L can be modeled with three thermal components at temperatures similar to those found in Liu et al. (2011); specifically, the two energized sub-populations have temperatures $T=2.1 m_{e} c^{2}$ and $T=3.5 m_{e} c^{2}$. Neither a power law nor the Bessho \& Bhattacharjee (2012) spectral form present a good fit to the spectrum in this simulation. It is important to note that the continued evolution of the particle energy spectrum we observe after the current sheet has become disordered, and the likely additional evolution that would be taking place in an even larger simulation, mean that the spectral form has not converged. For example, it is possible that in addition to the two thermal components that we have detected, additional such populations at still higher temperatures would appear in larger simulations, and that the combination of those would constitute a hard spectral tail, although it is uncertain that this tail would take the form of a power law.

In PIC simulations, distinguishing between the various forms of energized particle spectra is difficult due to dynamic range limitations. Another complication is how the spectrum of the non-energized, yet possibly adiabatically heated background plasma is to be subtracted to isolate the genuine nonthermal component. Insight can separately be gained by examining test particle trajectories in magnetic field geometries modeling reconnection layers. Particle ballistics in X-line as well as magnetic island geometries introduced at various levels of approximation has been investigated in numerous studies (e.g., Zenitani \& Hoshino 2001; Larrabee et al. 2003; Bessho \& Bhattacharjee 2012; Cerutti et al. 2012a). Often, the models entail transport terms describing particle acceleration while confined in a single $\mathrm{X}$-line region or an island, and other terms describing particle escape and the termination of acceleration. Then, the terminal energy spectrum of the escaping particles is obtained by taking the input energy spectrum and deterministically transforming it by the transport terms. The crudest such models have suggested that the terminal spectrum could be a power law, e.g., of the form $d N / d \gamma \propto \gamma^{\alpha}$ with $\alpha=-(2 / \pi) B_{\mathrm{rec}} / E_{\mathrm{RR}} \sim-(2 / \pi) c / v_{\mathrm{A}}$, where $B_{\text {rec }}$ is the reconnected magnetic field and $E_{\mathrm{RR}}$ is the electric field in the reconnection region discussed in Section 3.5 (Zenitani \& Hoshino 2001). Models taking a more detailed accounting of the transport of particle phase space coordinates and the kinematics of escape, however, can instead imply distinctly non-power-law, softer spectra (e.g., Bessho \& Bhattacharjee 2012). It is also important to note that the breaking of translational invariance in the direction of the current, e.g., by threedimensional phase decoherence (see Section 3.1), may limit particle acceleration and prevent the production of the same energized particle tail produced in two-dimensional geometries.

Power-law spectra are generically expected in acceleration processes entailing stochasticity. A prime example is the linear diffusive shock acceleration in which the random outcome of particle scattering in the shock downstream determines whether the particle will return to the shock upstream and be subjected to another acceleration cycle (Bell 1978; Blandford \& Ostriker 1978). In contrast, the acceleration in an idealized time-independent and two-dimensional X-line region that lacks substructure in the form of secondary and embedded islands, should be deterministic. Therefore, the models of Zenitani \& Hoshino (2001), Larrabee et al. (2003), and Bessho \& Bhattacharjee (2012) can be thought of as producing a powerlaw-like spectrum only "by coincidence." Realistic reconnection regions should plausibly allow particles to be accelerated in stochastic fashion as they contain time dependence and structure on multiple scales; they may also have a mechanism for returning particles that have escaped an acceleration site into another such site (see, e.g., Drake et al. 2006, 2010; Kowal et al. 2011; Hoshino 2012). Since in this work we have found that the reconnection layer transitions into a disordered network of interacting flux ropes, it will be particularly interesting to 
investigate, in subsequent studies, if this network allows for stochastic acceleration of particles in intermittent, secondary reconnection sites that may appear in the course of the flux rope network evolution.

\section{CONCLUSIONS}

In this paper, we carried out three-dimensional PIC simulations of magnetic reconnection in a relativistic pair plasma with varying guide field strength. Plasma magnetizations, expressed in terms of the magnetic-to-kinetic pressure ratio, were of the order of unity. The initial conditions differed from the usual Harris sheet configuration by not having a large density contrast between the center of the current sheet and the background plasma. We investigated the growth of unstable kinetic modes in the current sheet, as well as the nonlinear development of a three-dimensional flux rope network. We also investigated the character and efficiency of particle energization. Our main results can be summarized as follows.

The current sheets in all simulations develop significant magnetic reconnection accompanied by the conversion of magnetic to particle kinetic energy. With the aid of Fourier decomposition, we ascertained that in all runs but Run S1K0, the linear tearing mode is dominant in the early evolution of the current sheet, and no significant growth occurs in the linear kink and oblique modes. The nonlinear development of the tearing mode produces a chain of flux ropes separated by primary X-lines. The flux ropes merge in hierarchical fashion whereby the merging time scale is proportional to the flux rope separation. During this phase, magnetic reconnection takes place at the $\mathrm{X}$-lines. We find that the dimensionless reconnection rates $\sim(0.05-0.08)$ and the maximum outflow speeds $\approx 0.4 c \sim v_{\mathrm{A}} / 2$ in our simulations are similar to those detected in other three-dimensional simulations of reconnection in pair plasmas. We also find that spatial variation of an off-diagonal component of the pressure tensor is responsible for the breaking of flux freezing at the X-lines, consistent with existing results.

While the hierarchical flux rope merging process initially appears similar to that found in two-dimensional simulations, in fact it is three-dimensional from the outset. This is because a lack of initial phase coherence in the linear tearing mode on scales larger than those allowed by causality breaks translational invariance in the direction of the initial current flow. The flux ropes form a topologically interconnected, dynamically evolving network. Dynamical interaction between neighboring flux ropes is provided by magnetic tension forces. With time, the flux ropes break up into segments with more isotropic orientations. The strongly three-dimensional character of the reconnection layer seems to suggest that global reconnection models invoking quasi-two-dimensional plasmoid hierarchies (e.g., Shibata \& Tanuma 2001; Fermo et al. 2010; Uzdensky et al. 2010) require revision to account for the inter-plasmoid magnetic linkage and isotropization of plasmoid orientations.

The larger flux ropes produced during flux rope merging contain substructure down to plasma skin depth scales which is reflected in embedded, twisted and braided current filaments and sheets. Overall, this substructure is force-free and evolves relatively slowly. However, isolated sites within the evolved flux rope network contain spatially and temporally intermittent sites characterized by strong nonideal conditions $\mathbf{E} \cdot \mathbf{B} \neq 0$ where a change of magnetic connectivity continues to take place even after flux rope merging has saturated on length scales equal to the size of the computational box. This intermittency may produce the observed variability of nonthermal emission in systems in which the emitting particles are energized by magnetic reconnection.

During the early, ordered flux rope merging phase, particles are accelerated to high Lorentz factors by the electric field in primary X-lines; the trajectories of these particles are well described by Speiser orbits. Particles continue to be energized in the later, disordered phase we identify in our largest simulation, but we leave the analysis of energization in the disordered regime to a subsequent investigation.

Simulations with higher magnetization and lower guide field strength exhibit greater and faster energy conversion and particle energization. The efficiency of particle energization measured in terms of the energy in the accelerated particles per unit magnetic energy dissipated in the simulation is an increasing function of the guide field strength for $\sigma=1$, which can be interpreted as resulting from a decreasing plasma compressibility with increasing guide field. The final particle energy spectrum in the largest simulation is best fit by the inclusion of new thermal components at temperatures $2.1 m_{e} c^{2}$, and $3.5 m_{e} c^{2}$, in addition to the initial thermal component with temperature $m_{e} c^{2}$. We, however, acknowledge that a larger size or longer duration simulation is likely to produce a still more pronounced energized component, possibly even a population described with a power law spectrum.

Energetic positrons (electrons) with Lorentz factors $\gamma>30$ are moderately beamed in (opposite to) the direction of the initial current flow with median inclinations of $\sim 30^{\circ}-40^{\circ}$. The degree of beaming is determined by a particle's energy gain during acceleration. We speculate that more highly magnetized plasmas and reconnection sites with larger size X-line regions should give rise to stronger beaming.

In this work, we have investigated a narrow range of magnetizations with $\sigma \sim \mathcal{O}(1)$, but astrophysical reconnection sites can also have high magnetizations $\sigma \gg 1$. We can speculate about the applicability of our results in the latter limit. The linear tearing mode responsible for the initiation of reconnection is insensitive to the degree of magnetization far from the current sheet. The phase decoherence that produces the initial breakdown of translational invariance is determined by the tearing mode growth time and should thus also persist at high magnetizations. Therefore, we expect the qualitative structure of the reconnection region at higher values of $\sigma$ to be similar to that found in our simulations.

The primary effect of high magnetization is that the Alfvén velocity approaches the speed of light, which could give rise to ultrarelativistic outflows from the X-line region. In such outflows, the inertial term of the generalized Ohm's law becomes important in the breaking of flux freezing (Hesse \& Zenitani 2007). This in turn may increase the dimensionless reconnection rate $r_{\text {rec }}$ relative to the value found in our simulations. It remains to be seen whether the associated reconnection process is more or less intermittent. An increased magnetization is likely to increase the efficiency and the degree of beaming in particle energization.

We consider these results and the immediate questions they raise an incremental step in the development of a multiscale view of collisionless plasma self-organization during magnetic reconnection. Further work is clearly required to place our key finding, the evolution of the simulated, periodic reconnection layer into a disordered network of interacting magnetic flux ropes, in the macroscopic context of a realistic reconnection site characterized by outflow boundary conditions and altogether 
different field line asymptotics at large distances from the X-line. It will be particularly interesting to see if the reconnected-fluxcarrying outflow from the macroscopic X-line will possess the disordered, interlinked magnetic field topology we observe and investigate what will be the character of magnetic fluctuations in the outflow.

This work was initially inspired by Professor Pawan Kumar's reflections on the physics of prompt emission in gamma-ray burst sources and we would like to thank Professor Kumar for many stimulating conversations. D.K. would also like to thank Professors Richard Fitzpatrick and Wendell Horton for useful discussions. A.S. is supported by NSF grant AST-0807381 and NASA grants NNX09AT95G and NNX10A039G. The authors acknowledge the Texas Advanced Computing Center (TACC) at The University of Texas at Austin for providing HPC resources that have contributed to the research results reported within this paper. M.M. and A.S. also gratefully acknowledge support and hospitality from the Kavli Institute for Theoretical Physics during their workshop Particle Acceleration in Astrophysical Plasmas supported in part by the National Science Foundation under grant No. PHY05-51164. We thank the Computational Information Systems Laboratory at the National Center for Atmospheric Research for providing the VAPOR analysis tool (Clyne et al. 2007).

\section{REFERENCES}

Baalrud, S. D., Bhattacharjee, A., \& Huang, Y.-M. 2012, PhPl, 19, 022101 Bell, A. R. 1978, MNRAS, 182, 147

Berger, M. A. 1997, JGR, 102, 2637

Berger, M. A. 1999, PPCF, 41, B167

Bessho, N., \& Bhattacharjee, A. 2005, PhRvL, 95, 245001

Bessho, N., \& Bhattacharjee, A. 2007, PhPl, 14, 056503

Bessho, N., \& Bhattacharjee, A. 2010, PhP1, 17, 102104

Bessho, N., \& Bhattacharjee, A. 2012, ApJ, 750, 129

Blandford, R. D., \& Ostriker, J. P. 1978, ApJL, 221, L29

Cerutti, B., Uzdensky, D. A., \& Begelman, M. C. 2012a, ApJ, 746, 148

Cerutti, B., Werner, G. R., Uzdensky, D. A., \& Begelman, M. C. 2012b, ApJL, 754, L33

Che, H., Drake, J. F., \& Swisdak, M. 2011, Natur, 474, 184

Chen, L., Bhattacharjee, A., Puhl-Quinn, P. A., et al. 2008, NatPh, 4, 19

Clyne, J., Mininni, P., Norton, A., \& Rast, M. 2007, NJPh, 9, 301

Daughton, W. 1999, PhPl, 6, 1329

Daughton, W., \& Karimabadi, H. 2007, PhPl, 14, 072303

Daughton, W., Roytershteyn, V., Karimabadi, H., et al. 2011, NatPh, 7, 539

Drake, J. F., Opher, M., Swisdak, M., \& Chamoun, J. N. 2010, ApJ, 709, 963

Drake, J. F., Swisdak, M., Che, H., \& Shay, M. A. 2006, Natur, 443, 553

Egedal, J., Daughton, W., Drake, J. F., Katz, N., \& Lê, A. 2009, PhP1, 16, 050701

Egedal, J., Daughton, W., \& Le, A. 2012, NatPh, 8, 321
Fermo, R. L., Drake, J. F., \& Swisdak, M. 2010, PhPl, 17, 010702

Fu, X. R., Lu, Q. M., \& Wang, S. 2006, PhPl, 13, 012309

Giannios, D. 2010, MNRAS, 408, L46

Hesse, M., Neukirch, T., Schindler, K., Kuznetsova, M., \& Zenitani, S. 2011, SSRv, 160, 3

Hesse, M., \& Zenitani, S. 2007, PhPl, 14, 112102

Hoshino, M. 2012, PhRvL, 108, 135003

Hoshino, M., Mukai, T., Terasawa, T., \& Shinohara, I. 2001, JGR, 106, 25979

Huang, C., Lu, Q., \& Wang, S. 2010, PhPl, 17, 072306

Jaroschek, C. H., Treumann, R. A., Lesch, H., \& Scholer, M. 2004, PhPl, 11,1151

Kowal, G., de Gouveia Dal Pino, E. M., \& Lazarian, A. 2011, ApJ, 735, 102

Krall, N. A., \& Trivelpiece, A. W. 1973, Principles of Plasma Physics (New York: McGraw-Hill)

Larrabee, D. A., Lovelace, R. V. E., \& Romanova, M. M. 2003, ApJ, 586, 72

Liu, W., Li, H., Yin, L., et al. 2011, PhP1, 18, 052105

Lyubarsky, Y., \& Kirk, J. G. 2001, ApJ, 547, 437

Lyubarsky, Y., \& Liverts, M. 2008, ApJ, 682, 1436

Lyutikov, M. 2003, MNRAS, 346, 540

Markidis, S., Henri, P., Lapenta, G., et al. 2012, NPGeo, 19, 145

McKinney, J. C., \& Uzdensky, D. A. 2012, MNRAS, 419, 573

Nalewajko, K., Giannios, D., Begelman, M. C., Uzdensky, D. A., \& Sikora, M. 2011, MNRAS, 413, 333

Øieroset, M., Phan, T. D., Eastwood, J. P., et al. 2011, PhRvL, 107, 165007

Oka, M., Fujimoto, M., Shinohara, I., \& Phan, T. D. 2010a, JGR, 115, A08223

Oka, M., Phan, T., Krucker, S., Fujimoto, M., \& Shinohara, I. 2010b, ApJ, 714, 915

Parfrey, K., Beloborodov, A. M., \& Hui, L. 2012, ApJL, 754, L12

Priest, E., \& Forbes, T. 2000, Magnetic Reconnection (Cambridge: Cambridge Univ. Press)

Pritchett, P. L. 2008, PhPl, 15, 102105

Roytershteyn, V., \& Daughton, W. 2008, PhPl, 15, 082901

Schindler, K., Hesse, M., \& Birn, J. 1988, JGR, 93, 5547

Schmitz, H., \& Grauer, R. 2006, PhPl, 13, 092309

Shibata, K., \& Tanuma, S. 2001, EP\&S, 53, 473

Sironi, L., \& Spitkovsky, A. 2011, ApJ, 741, 39

Speiser, T. W. 1965, JGR, 70, 4219

Spitkovsky, A. 2008, ApJL, 673, L39

Takahashi, H. R., Kudoh, T., Masada, Y., \& Matsumoto, J. 2011, ApJL, 739, L53

Tanaka, K. G., Fujimoto, M., Badman, S. V., \& Shinohara, I. 2011, PhP1, 18,022903

Thompson, C., \& Duncan, R. C. 1995, MNRAS, 275, 255

Uzdensky, D. A., Cerutti, B., \& Begelman, M. C. 2011, ApJL, 737, L40

Uzdensky, D. A., Loureiro, N. F., \& Schekochihin, A. A. 2010, PhRvL, 105,235002

Vasyliunas, V. M. 1975, RvGSP, 13, 303

Volponi, F., Yoshida, Z., \& Tatsuno, T. 2000, PhPl, 7, 2314

Yamada, M., Kulsrud, R., \& Ji, H. 2010, RvMP, 82, 603

Yin, L., Daughton, W., Karimabadi, H., et al. 2008, PhRvL, 101, 125001

Zenitani, S., \& Hesse, M. 2008, ApJ, 684, 1477

Zenitani, S., \& Hoshino, M. 2001, ApJL, 562, L63

Zenitani, S., \& Hoshino, M. 2005, PhRvL, 95, 095001

Zenitani, S., \& Hoshino, M. 2007, ApJ, 670, 702

Zenitani, S., \& Hoshino, M. 2008, ApJ, 677, 530

Zhang, B., \& Yan, H. 2011, ApJ, 726, 90 